To appear in AJ

\title{
A Comprehensive, Wide-Field Study of Pulsating Stars in the Carina Dwarf Spheroidal Galaxy
}

\author{
A. Katherina Vivas \\ Centro de Investigaciones de Astronomía (CIDA) \\ Apartado Postal 264, Mérida 5101-A, Venezuela \\ akvivas@cida.ve \\ and \\ Mario Mateo \\ Department of Astronomy, University of Michigan \\ 500 Church St. Ann Arbor, MI 48109, USA \\ mmateo@umich.edu
}

\begin{abstract}
We report the detection of 388 pulsating variable stars (and some additional miscellaneous variables) in the Carina dSph galaxy over an area covering the full visible extent of the galaxy and extending a few times beyond its photometric (King) tidal radius along the direction of its major axis. Included in this total are 340 newly discovered dwarf Cepheids which are mostly located $\sim 2.5$ magnitudes below the horizontal branch and have very short periods $(<0.1$ days) typical of their class and consistent with their location on the upper part of the extended main sequence of the younger populations of the galaxy. Several extra-tidal dwarf cepheids were found in our survey up to a distance of $\sim 1^{\circ}$ from the center of Carina. Our sample also includes RR Lyrae stars and anomalous Cepheids some of which were found outside the galaxy's tidal radius as well. This supports past works that suggests Carina is undergoing tidal disruption. We use the periodluminosity relationship for dwarf Cepheids to estimate a distance modulus of $\mu_{0}=20.17 \pm 0.10$ mags, in very good agreement with the estimate from RR Lyrae stars. We find some important differences in the properties of the dwarf
\end{abstract}


Cepheids of Carina and those in Fornax and the LMC, the only extragalactic samples of dwarf Cepheids currently known. These differences may reflect a metallicity spread, depth along the line of sight and/or, different evolutionary paths of the dwarf Cepheid stars.

Subject headings: galaxies: dwarf, galaxies: individual(Carina), galaxies: stellar contents, Local Group, stars: variables: general

\section{Introduction}

The dwarf spheroidal (dSph) galaxies surrounding the Milky Way have proven to be complex objects with extraordinary, and different, star formation histories (e.g. Mateo 1998; Grebel 2011). No two dwarf galaxies in our neighbourhood are alike and the reason(s) why these galaxies present such a variety of star formation and chemical enrichment histories is not well known today. The understanding of the nature and evolution of these systems is fundamental for studying their role in the hierarchical process of formation of large galaxies like our own Milky Way.

At $\sim 100 \mathrm{kpc}$ of distance, Carina is one of the best known of the "classical" dSph galaxies, in large part because of the rather extraordinary characteristics of its stellar populations. For example, the galaxy presents a rich color-magnitude diagram (CMD) in which it is clear the existence of multiple stellar populations of ages around 11, 5 and 1 Gyr (Smecker-Hane et al. 1996; Hurley-Keller et al. 1998; Monelli et al. 2003). The CMD of Carina - in particular its very narrow red giant branch - suggests that the spread in metallicity should be small in this galaxy (e.g. Rizzi et al. 2003; Bono et al. 2010), though the well-known degeneracy between age and metallicity coupled with the internal age spread in Carina may be conspiring to mimic a small abundance spread. Spectroscopic measurements seem to show inconclusive and somewhat contradictory results on this issue (Koch et al. 2006; Helmi et al. 2006). In addition, Carina appears to be suffering significant tidal erosion from its interactions with the Milky Way as suggested from the existence of of Carina members beyond its photometric (King 1962) tidal radius (Majewski et al. 2000; Muñoz et al. 2006; Battaglia et al. 2012).

Pulsating stars have been frequently used to study the stellar population in stellar systems. The presence of RR Lyrae stars, for example, is an unequivocal signal that an old population (>10 Gyrs) in the system exists. On the other hand, anomalous Cepheids, which are common in dSph galaxies but not in galactic globular clusters (Nemec et al. 1994; Clement et al. 2001), are usually interpreted as belonging to an intermediate/young stellar population (Zinn \& Searle 1976; Bono et al. 1997; Dall'Ora et al. 2003; Kuehn et al. 2008; 
Kinemuchi et al. 2008; Fiorentino et al. 2012, among others). Both types of pulsating stars have been found already in Carina (Saha et al. 1986; Dall'Ora et al. 2003), consistent with what is know about the overall properties of its stellar populations.

Below the horizontal branch, the instability strip crosses an interesting location in the CMD of Carina where pulsating stars from different evolutionary paths may coexist. There are not only stars from the young and the intermediate age main sequence but also blue stragglers from the old population of the galaxy. The nomenclature of the pulsating stars in this part of the instability strip is confusing. We will follow Mateo (1993) and will refer to them as dwarf Cepheids (DC). Some recent works have preferred to name them either as $\delta$ Scuti or as SX Phe depending on they belonging to a Population I or II, respectively. In complex systems such as Carina, the concepts of Pop I and II are not so easy to apply as it is not straightforward to determine the metallicities of the population(s) in the region of the instability strip. Consequently, based on common definitions of the classes, a dwarf galaxy such as Carina could contain both $\delta$ Scuti and SX Phe stars though the data to distinguish them may not be readily available for the foreseeable future. For all these reasons, the collective name 'dwarf Cepheid' seems more appropriate when applied to variables in objects like Carina.

Properties of this kind of variable stars are discussed extensively in Breger (2000). The key practical property of these stars is that DCs are short period variables $(\sim 0.03$ to 0.25 days). As a result, any observational study of these stars must maintain a short cadence while maintaining sufficient signal-to-noise to generate useful light curves for detailed discovery, classification and analysis. Fortunately, SX Phe and a sub-group of the $\delta$ Scuti stars named HADS (high amplitude Delta Scuti) have large amplitudes, amounting to several tenths of a magnitude, making these objects feasible targets with medium-size telescopes even at the distance of a galaxy such as Carina.

Mateo et al. (1998) presented the first detections of DCs in Carina. They studied three small fields near the center of Carina and detected 20 of these short-period pulsators. Since DC stars obey a period-luminosity relationship, Mateo et al. (1998) derived a distance to Carina which was in very good agreement with estimates based on other types of variables and the properties of the galaxy's red giant branch. Given the pronounced mixture of stellar populations in Carina and, in particular, the importance of its intermediate age population, Mateo et al. (1998) speculated that the observed sample of DCs was likely just a small fraction of a likely large population of DCs in the galaxy. In this work we extend the previous observations by Mateo et al. (1998) and search for DC in a much larger area, covering not only the whole galaxy but extending up to several tidal radii along the direction of the semi-major axis. Our aim is to use the DCs as possible tracers of extra-tidal features in 
Carina and, if found in sufficient quantities, as possible probes of the line-of-sight depth of the galaxy. RR Lyrae stars and anomalous Cepheids, which appeared naturally in our data, can also trace the extended structures of Carina.

We report here the discovery of 340 DC stars in Carina, which constitute one of the largest extra-galactic samples of this type of stars known to date. Besides Carina, DCs have also been found in large numbers only in two other extragalactic systems, namely, Fornax (Poretti et al. 2008) and the LMC (Garg et al. 2010). The different combinations of age and metallicity of the stellar populations in these galaxies allow us for the first time to compare the properties of DCs in distinct stellar systems. Such comparisons are useful not only to shed light on the origin and specific frequency of DCs under different environments, but also, to study their use as standard candles and as possible tracers of the 3D structure of galaxies.

\section{Observational strategy and data reduction}

Multi-epoch observations of eight fields around the Carina dSph galaxy were taken with the MOSAIC-II camera at the $4 \mathrm{~m}$ Blanco Telescope at Cerro Tololo Interamerican Observatory (CTIO), Chile, during an interval of 6 consecutive nights in December 2007. Table 1 shows the central coordinates of the observed fields, the number of nights in which each field was observed and the total number of observations in the B and $\mathrm{V}$ bands. The complete survey covers the whole of the Carina dSph galaxy out to its classical (King 1962) tidal radius of 28.8 (Irwin \& Hatzidimitriou 1995) and extends up to $\sim 1.5$ from the galaxy center in each direction along its semi-major axis. The outermost surveyed regions are located at about three tidal radii from the galaxy center.

Since the main goal of these observations was to identify short period variables $(<0.25$ d), the basic approach consisted in obtaining repeated observations of the same field during a night. In general, continuous observations of the same field for a period of $\sim 3-4$ hours was obtained, typically alternating exposures in B and V. This strategy was chosen to span a full pulsation cycle of the longest-period DC variables anticipated to be present in Carina. In practice, the multi-hour sequences covered one complete pulsation cycle for stars with periods $(\lesssim 0.15 \mathrm{~d})$, and multiple $(2-3)$ pulsation cycles for the majority of DC which are expected to have periods of the order of only 1 hour $(\sim 0.05) \mathrm{d})$. Additional observations of each field were taken as well in different nights to extend the temporal coverage over the full extent of the run. Figure 1 shows the total number of observations $\left(N_{B}+N_{V}\right)$ in the whole survey region. Of these, $\sim 60 \%$ are $\mathrm{V}$ observations and the rest are B. Fields had a sizeable overlap resulting in some regions having a larger number of observations. Fields 7 and 8 were observed only twice in each band. Only the parts of those two fields overlapping 


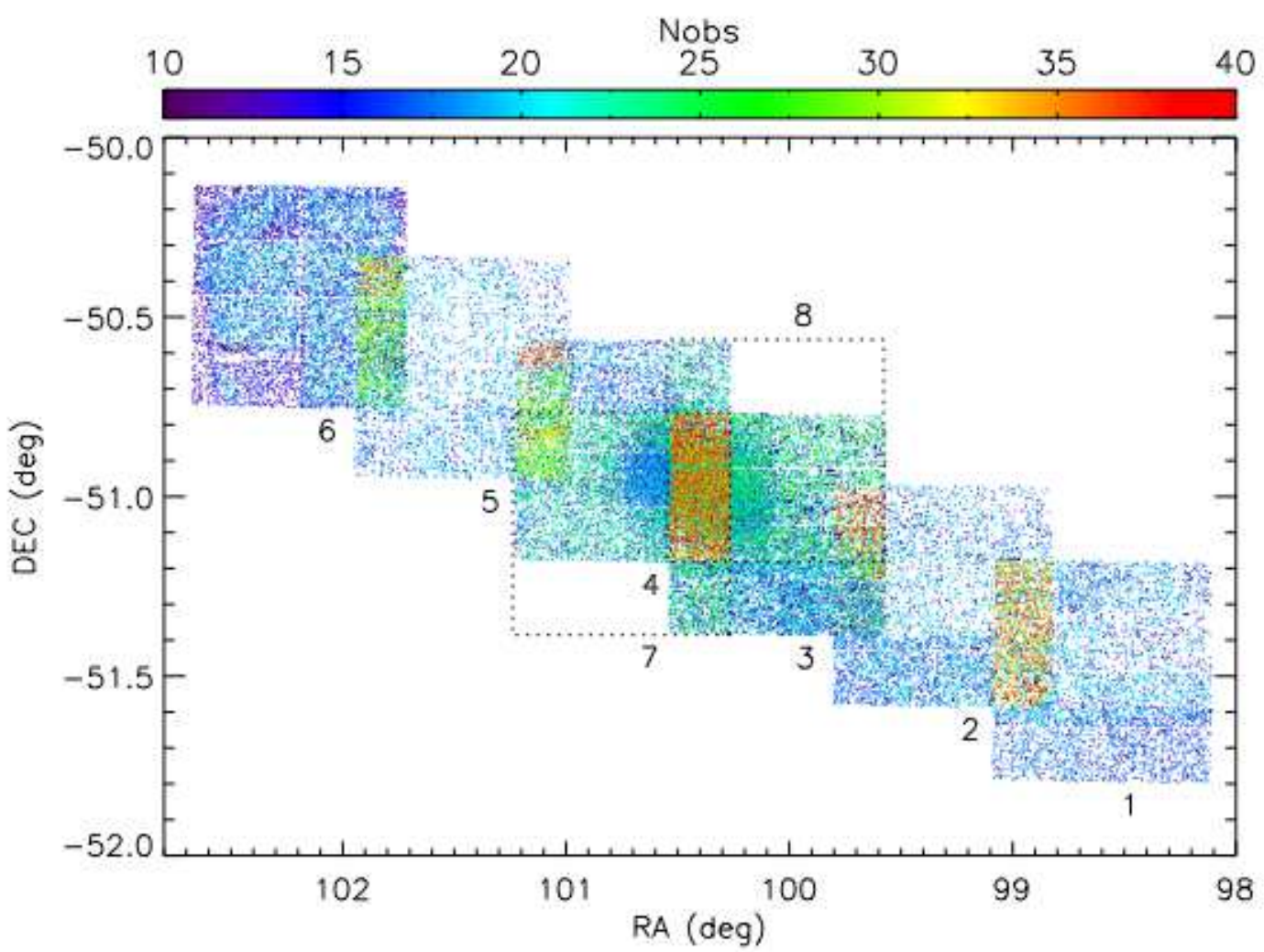

Fig. 1. - Total number of observations $\left(N_{o b s}=N_{V}+N_{B}\right)$ in all the survey region. Numbers correspond to the Field number according to Table 1 . Fields 7 and 8, which had very few observations, are shown with dotted lines.

other fields were used in this work. There are at least 20 observations (total, in B and V) per star in all the region although the central parts of the galaxy have significantly more observations, in some cases approaching 50 or more epochs. Depending on seeing conditions, exposure times varied from $480 \mathrm{~s}$ to $600 \mathrm{~s}$ in $\mathrm{V}$ and $500 \mathrm{~s}$ to $600 \mathrm{~s}$ in $\mathrm{B}$, corresponding to about $10 \%$ of the pulsation cycle of stars with periods of $0.05 \mathrm{~d}$. This dataset is the same one used recently by Battaglia et al. (2012) to determine an age gradient in Carina.

Individual frames were bias subtracted along rows using a constant derived from the image overscan region. Flat-field images were obtained using dome and twilight observations (suitably dithered to avoid stellar contamination) and supplemented by dark-sky flats obtained from our observations of the off-center fields in Carina. The latter were used only to apply very low spatial frequency corrections to post twilight/dome flattened images.

The photometry was carried out using DoPhot (Schechter et al. 1993). These reductions 
were carried out independently for each frame but using input coordinates derived from photometry of the stacked images. This resulted in more consistent photometric stability for non-variables and more uniform photometric catalogs for individual frames than reducing each frame independently. A typical distribution of photometric error as a function of $\mathrm{V}$ and $\mathrm{B}$ magnitudes is shown in Figure 2. We estimate that our data are typically complete to $\mathrm{V}, \mathrm{B} \sim 24.2$ since the number of detected sources drops for fainter magnitudes (Fig 2). Saturations signs start to be seen for magnitudes brighter than $B, V \sim 17$.

From the photometry of the individual frames we were able to to obtain time series for all stars in the region.

\section{Selection of variable stars}

The MOSAIC-II camera consists of eight $2048 \times 4096$ CCDs in a $4 \times 2$ array to cover a nearly square $36 \operatorname{arcmin}^{2}$ field. In order to ensure the best relative photometry in the time series for each star we treated each CCD individually. For each CCD, in each field, and each band, we selected a reference image, usually the one with the best seeing and hence the largest number of detected stars. Based on their celestial coordinates, we then matched stars in the reference image with all other observations not only from the same field but also from overlapping regions in other fields. A zero point difference was estimated after applying an iterative $3 \sigma$ clipping using all stars with photometric errors $<0.05$ mags. The $3 \sigma$ clipping avoided variable objects or spurious measurements to enter in the calculation of the zero point, which was then applied to all stars in each image. Even in images with only partial overlap with the reference image, the number of stars entering in the calculation of the zero point was large enough (usually over 100) to make this value very robust. This normalization procedure resulted in reliable time series for all stars in the fields from which we could then investigate variability.

Figure 3 shows the resulting standard deviation of the B and V magnitudes of stars for one of the fields (Field 3, CCD 7) which covers the innermost, hence most crowded, parts of Carina. The photometric error at the bright end amounts to $\sim 0.02$ and 0.03 mags in the $\mathrm{B}$ and $\mathrm{V}$ band, respectively. These numbers go down to $\sim 0.01 \mathrm{mag}$ in the outermost

fields where crowding is far less of an issue. For the stars of interest, the faint limit is similar in both photometric bands. Stars have in average a standard deviation of 0.1 mag at magnitude 23.8 in both bands. The main locus of stars shown in Figure 3 (and similar ones for others fields/CCDs) empirically define the photometric errors as a function of magnitude and may be compared to the photometric errors estimated by DoPhot in Figure 2. This run of photometric uncertainties for non-variable stars will be assumed for all stars from all 

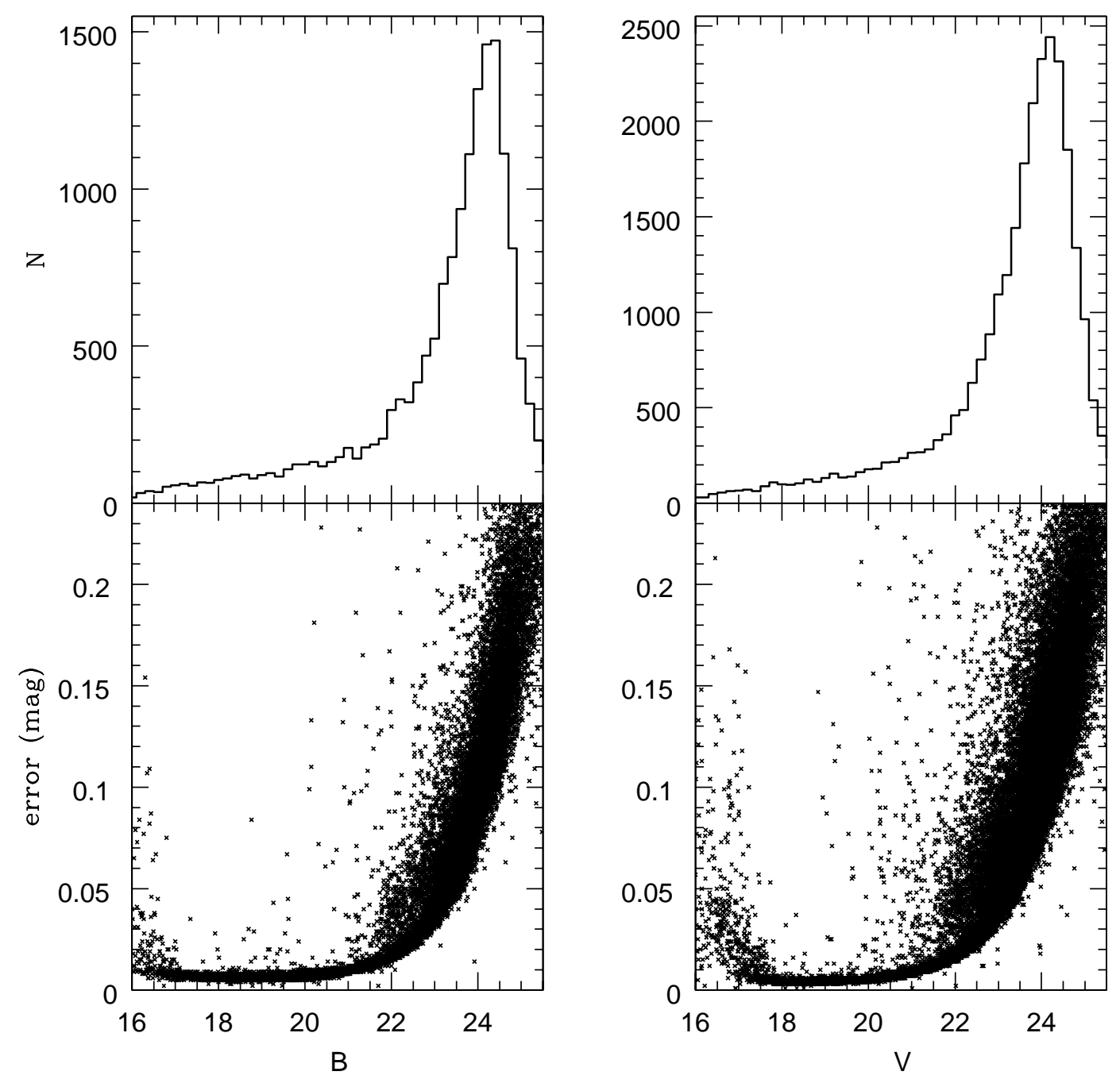

Fig. 2.- (Top) Number of sources detected in individual B and V images of Field 1 as a function magnitude. (Bottom) Errors given by DoPhot for stars in the same images. 
fields analysed in this study.

To identify variable objects we performed $\chi^{2}$ tests on each star independently for both the $\mathrm{B}$ and $\mathrm{V}$ bands. With this test we could flag as variable stars those stars whose variations in brightness are not likely due to photometric errors alone, by requesting that the probability distribution function be a small number, $P\left(\chi^{2}\right)<0.01$ (see for example Vivas et al. 2004; Watkins et al. 2009). The flagged stars are shown in the example field of Figure 3 as crosses. We required that stars should be flagged as variable in both $\mathrm{B}$ and $\mathrm{V}$ bands, a condition that eliminated many spurious or otherwise marginal cases.

Figure 4 shows the CMD in one of the central fields in Carina (Field 3, CCD 7) and one of the outermost ones (Field 1, CCD 7). Variable stars identified with the method just described are shown with $\times$ symbols. The upper panel clearly shows the very well known features of Carina: a double turnoff, a clear horizontal branch and a prominent red clump. It is also obviously apparent from this diagrams that Carina has a large population of variables. In particular, it is remarkable the large concentration of variable stars located in the upper main sequence, all of which are clear candidate DC variables. There are also variable objects at the horizontal branch, which are expected to be RR Lyrae stars. Brighter blue variables may be anomalous Cepheids known to exist in the Carina stellar population (Saha et al. 1986; Nemec et al. 1994; Dall'Ora et al. 2003). In the particular field shown in the upper panel of Figure $4,602(\sim 4 \%)$ of the stars were flagged as variable using our $\chi^{2}$ criteria described above. The field in the lower panel is located at $\sim 2^{\circ}$ in RA from the center of Carina (Field 1, CCD7). The features of the galaxy are not easily discernible here due to the low number of stars (only the area covered by one CCD is shown). Previous studies have shown, however, that the galaxy appears to extend this far (Majewski et al. 2000; Muñoz et al. 2006; Battaglia et al. 2012, among others). The number of variables is lower in this field but nonetheless there are several ones located within the color-magnitude range more clearly defined by the variables at the center of the Carina.

Pulsating stars are expected to be located exclusively within their respective instability strip. To pare down the sample to include the most viable pulsating variable candidates, we have restricted our search for periodic variability to those stars with mean photometric properties that place them within a generous color range enclosed by the dotted lines in Figure $4(0<(B-V)<0.6)$. These limits in color are loose enough to give room for photometric errors and possible variable extinction. 

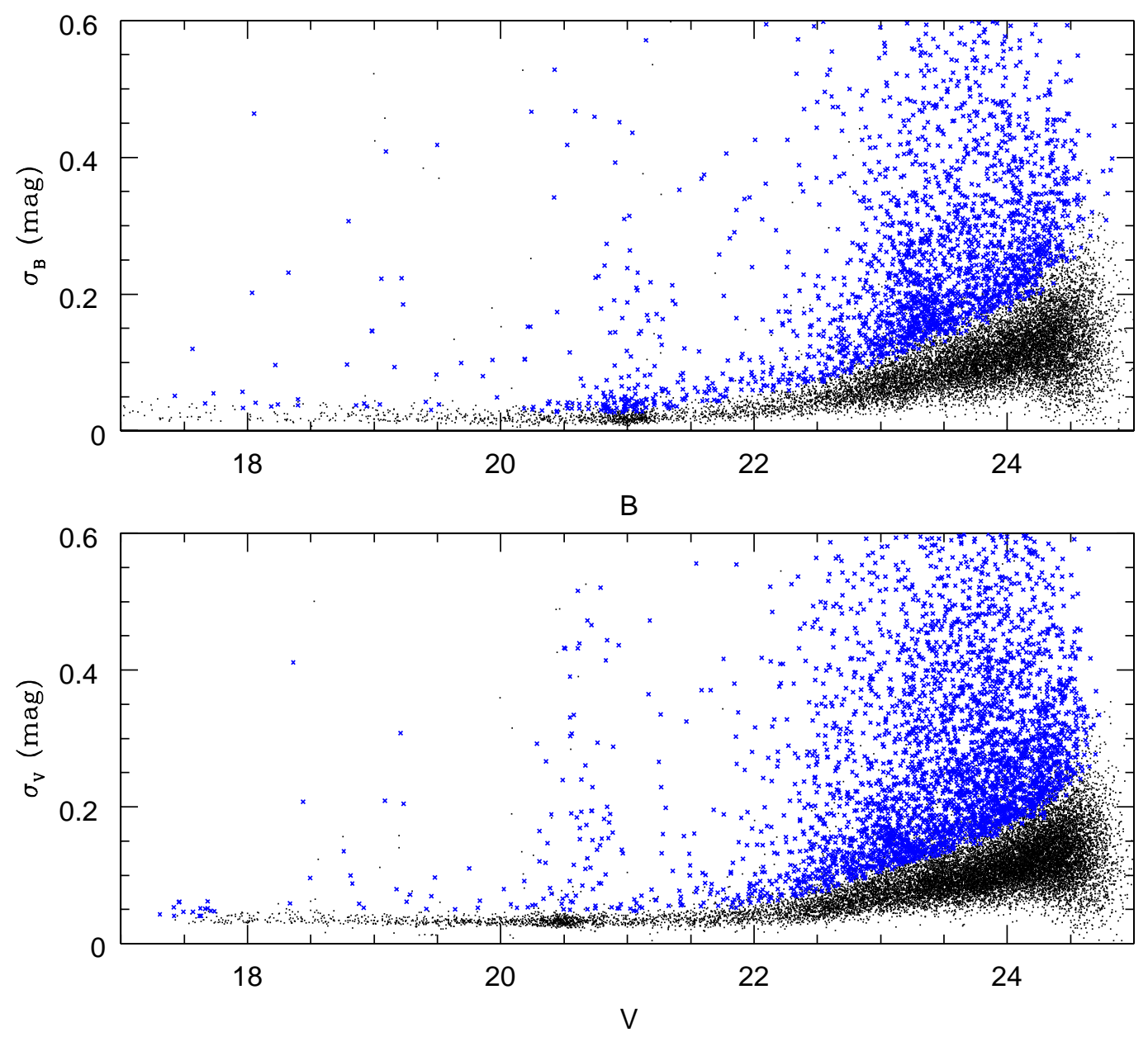

Fig. 3.- Standard deviation of the magnitudes of all stars in Field 3, CCD7, in B (top) and V (bottom) bands. This particular frame contains stars in the innermost part of the Carina dSph. Each star was observed up to 22 times in B and 30 times in V. Crosses in both diagrams indicate stars selected as variable by the $\chi^{2}$ test. 


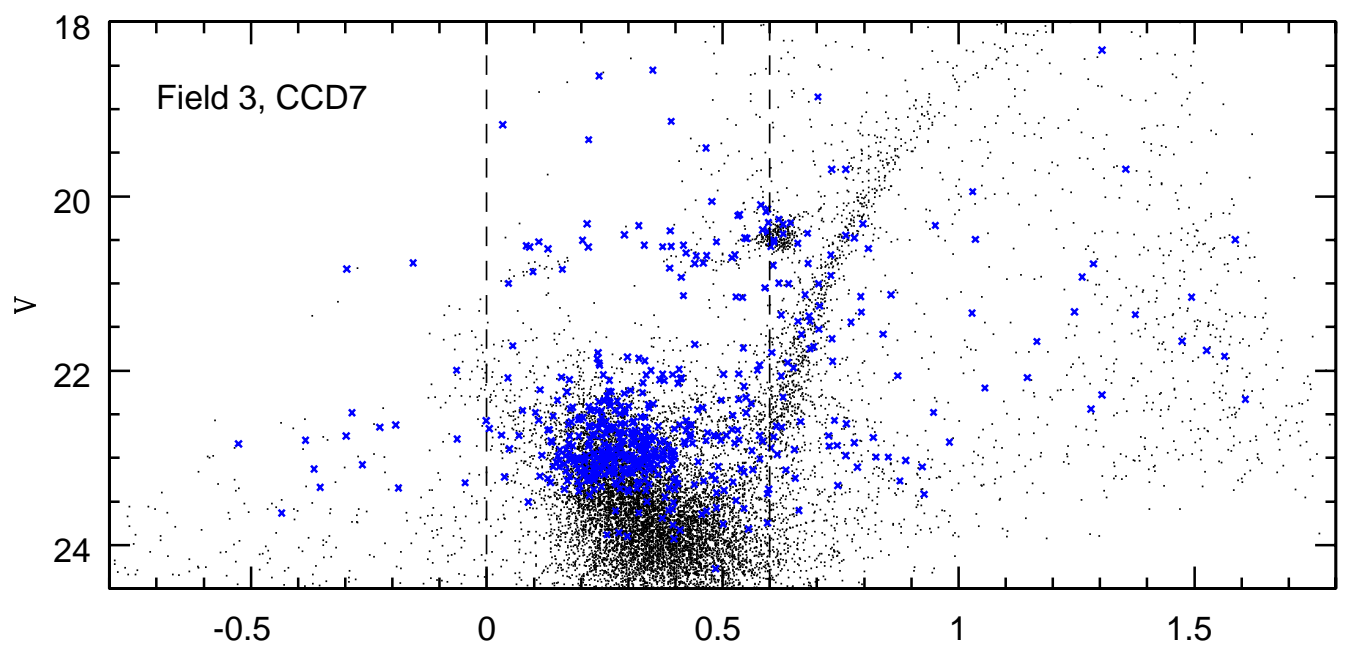

(B-V)

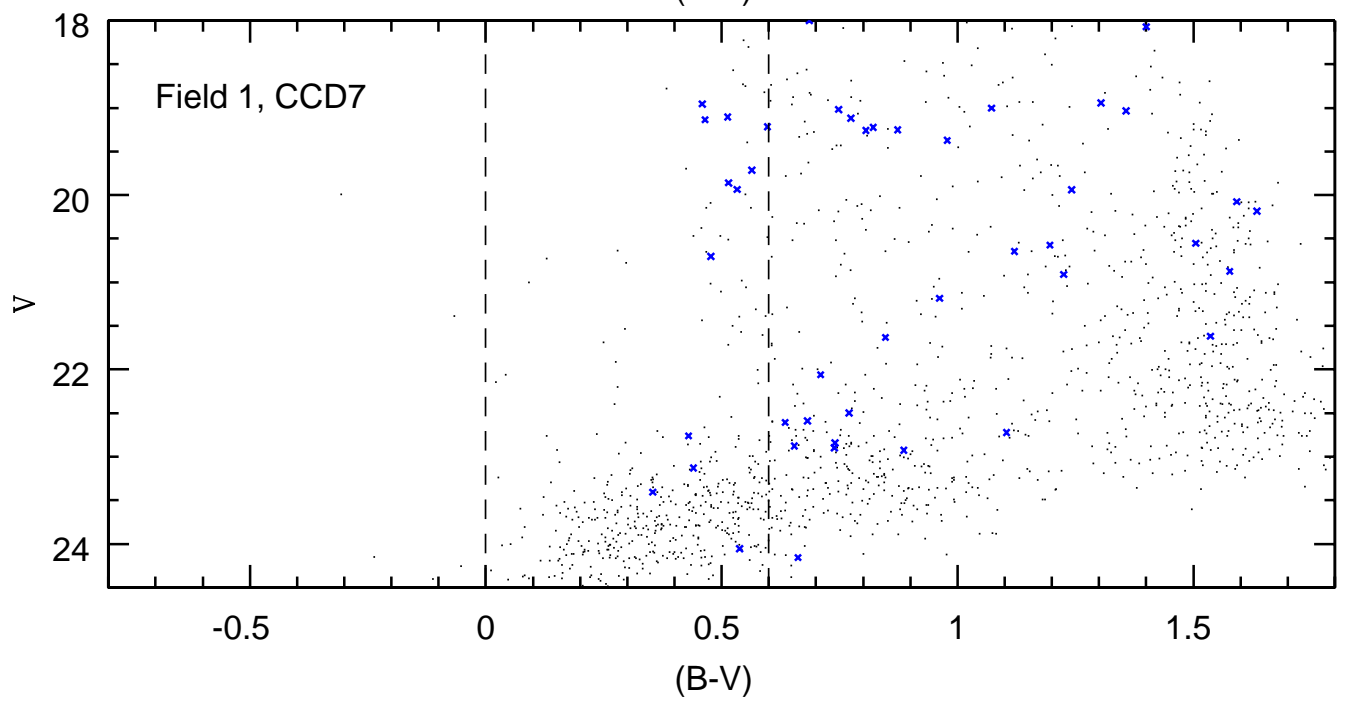

Fig. 4.- CMD in one of the fields near the center of Carina (top) and one outer field (bottom). Variable stars are shown as crosses. Variable stars within the dotted vertical lines were checked for periodicity. 


\section{Periodic variables}

The next step for the correct identification of the variable stars is the determination of periodicity. Our dataset does not have a large number of epochs which may be a problem to determine periods, though this is somewhat mitigated by the multi-hour (and multi-period, for DCs) stretches of observations in our dataset for each field. We used the combined B and V observations in conjunction with the Lafler \& Kinman (1965) algorithm. For each star we calculated the string length for a given trial period, defined by Lafler \& Kinman (1965) as:

$$
\Theta(\lambda)=\frac{\sum_{i}\left(m_{i}-m_{i+1}\right)^{2}}{\sum_{i}\left(m_{i}-\bar{m}\right)^{2}}
$$

where the magnitudes $m_{i}$ have been sorted by increasing phase for that particular trial period. If a star is periodic, the string length in both $\mathrm{B}$ and $\mathrm{V}$ bands should be a minimum when the data is phased with the correct period. For each trial period we calculated the string length in each band and then combined them weighting by the number of observations in each band (Stetson 1996; Watkins et al. 2009; Mateu et al. 2012):

$$
\Theta=\frac{N_{V} \Theta(V)+N_{B} \Theta(B)}{N_{V}+N_{B}}
$$

We imposed a requirement of a minimum of 5 observations in each band, and at least 15 observations in total $\left(N_{V}+N_{B}\right)$ to use this approach to test a star for periodic variablity. To be able to study possible aliasing, for each star we selected the first three minima of $\Theta$ in the trial range. We checked visually the phased light curves of a star with these three periods if the parameter $\Lambda>2.0$. This parameter is defined in Lafler \& Kinman (1965) as the ratio between the string length away from and at the right period. The larger this number, the deeper the minimum and the most likely for the period to be real. The value of 2.0 was chosen arbitrarily after examining the results for a few fields so that we included a manageable number of false positives in our sample while minimizing the number of missed true periodic variables. The constraint is loose enough to allow the recognition of periodic variables even in stars for which a small number of epochs are available. If the trial period is the right one, the light curve should look smooth and coherent in both B and V bands. Since our main goal was to explore the faint pulsating variables we first searched for periodicity in the range 0.03 to $0.2 \mathrm{~d}$.

The bright variables in Carina have been explored extensively by Saha et al. (1986) and Dall'Ora et al. (2003, hereafter D03), although the latter in a region smaller than the one studied in this work. Our time sampling is not ideal for exploring periods $>0.5 \mathrm{~d}$ like the 
ones for RR Lyrae stars or anomalous Cepheids. Nonetheless, we tried the period search also in the range 0.2 to $0.9 \mathrm{~d}$, knowing that the completeness, due to our time sampling, may be low for stars in this range of periods.

Visual examination of the resulting light curves revealed aliasing affects in many of the short period variables. Indeed, for $\sim 1 / 3$ of the short period variables $(<0.02 \mathrm{~d})$, we found reasonable light curves with two different periods. According to Lafler \& Kinman (1965), the most common alias periods $(\Pi)$ are found when $p=1,1 / 2,2$ in the equation:

$$
\Pi^{-1}=P^{-1} \pm \frac{1}{p}
$$

where $P$ is the true period of the star. Figure 5 shows the relation between the two periods found for some stars in our sample. We also plotted the lines of the \pm 1 -day alias $(p=1$, solid line), the 2-day, and 1/2-day aliases ( $p=2$ and $1 / 2$, dashed and dotted lines respectively) given by equation 3. It is clear that the majority of the stars lie along the solid lines, confirming that the most common alias in our data is, as expected, the \pm 1 -day alias. The other two aliases are nonetheless also present. However, at the short period range of these stars, aliasing it is not a significant problem for our analysis since the differences between the real periods and their aliases are generally quite small, typically $<6 \%$. For the remainder of this paper we assumed the true period to be the one giving the best light curve to the eye, which is usually the one with the largest value of $\Lambda$. Nonetheless, we report all suspected aliases as well.

We found a total 397 periodic variable stars, in the range from 0.03 to $0.9 \mathrm{~d}$, in Carina. Based on their location on the CMD (Figure 6), and their periods and light curve shapes, we separated the periodic variables into four different groups which are seen in Figure 6 with different symbols. The majority of the periodic variable (340) are strongly concentrated in the upper part of the main sequence (+ symbols). We take these stars to be DCs which may encompass both SX Phe and large-amplitude $\delta$ Scuti stars. There are also many number of stars (38) at the level of the horizontal branch which we confidently presume to be RR Lyrae stars. At even brighter magnitudes we found 10 periodic variables which we identify as anomalous Cepheids, which have been known to exist in Carina (Saha et al. 1986, D03). There is finally a group of 9 stars whose identification is more uncertain an we classify as miscellaneous. Each one of these groups are discussed with more detail in the following sub-sections. Examples of light curves are shown in Figure 7. Because of the large number of variables, the full set of light curves is available as online-only material (Appendix A).

The period distributions of all variable stars are shown in Figure 8. DCs, by far the most numerous of the sample, were found to have periods between 0.03 and $0.18 \mathrm{~d}$, strongly peaked 


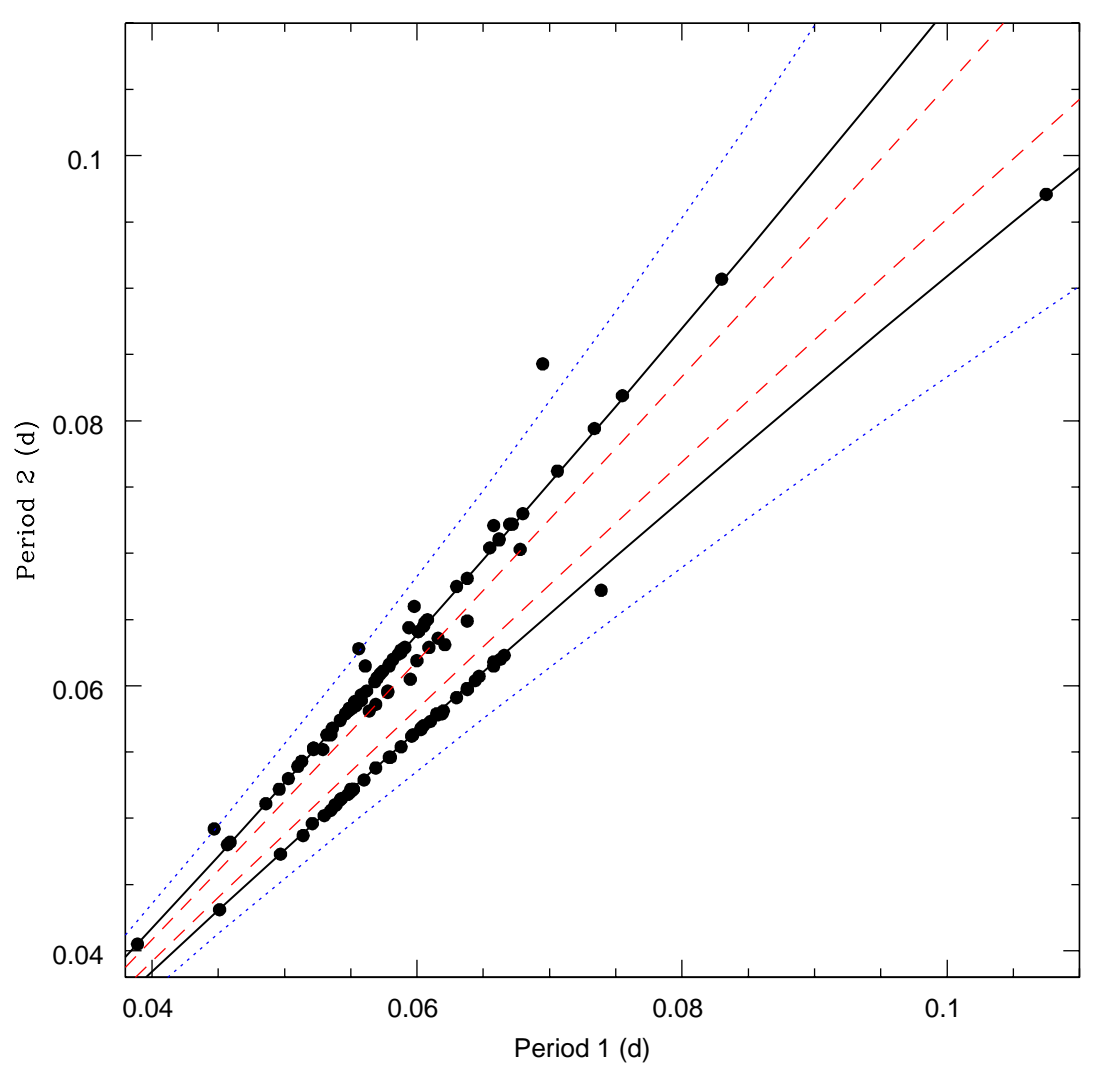

Fig. 5.- Relationship between the two possible periods found for 116 short-period variables. The lines show the expected relation given by equation 3 for $p=1$ (solid lines), $p=2$ (dashed lines) and $p=1 / 2$ (dotted lines).

at a period of $0.06 \mathrm{~d}$. Both RR Lyrae and anomalous Cepheid stars span a period range between 0.1 and $0.9 \mathrm{~d}$. Finally, the stars in the miscellaneous group, which are dispersed in the CMD but below the horizontal branch, have (uncertain) periods longer than the DC stars, ranging from 0.14 to $0.24 \mathrm{~d}$.

\subsection{RR Lyrae Stars}

We detected 38 RR Lyrae stars in our fields, 27 of them were classified as type $a b$ (RRab) and 11 as type $c$ (RRc). Their properties are listed in Table 2. Examples of the light curves of 2 RRab (left and middle column) and 1 RRc (right column) are shown in the second row of Figure 7. As mentioned before, D03 searched exhaustively for bright variables in Carina. 


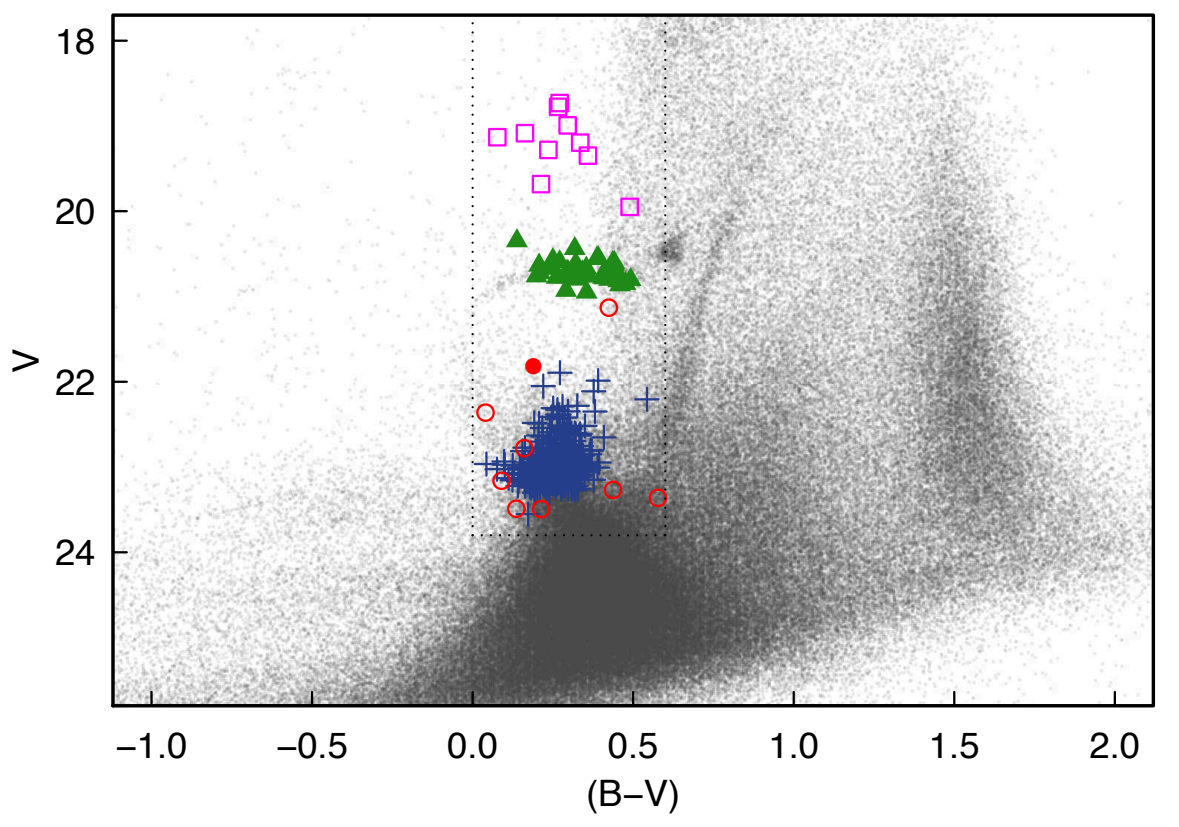

Fig. 6. - CMD of all stars in the survey. The colored symbols indicate the periodic variables found in this study: dwarf cepheids (+ symbols), RR Lyrae stars (solid triangles), anomalous cepheids (open squares) and miscellaneous stars (circles). Among the latter, the solid circle indicates the location of a clear case of an eclipsing binary. The dotted lines enclose the region of the CMD in which we search for periodicity among the variables. 

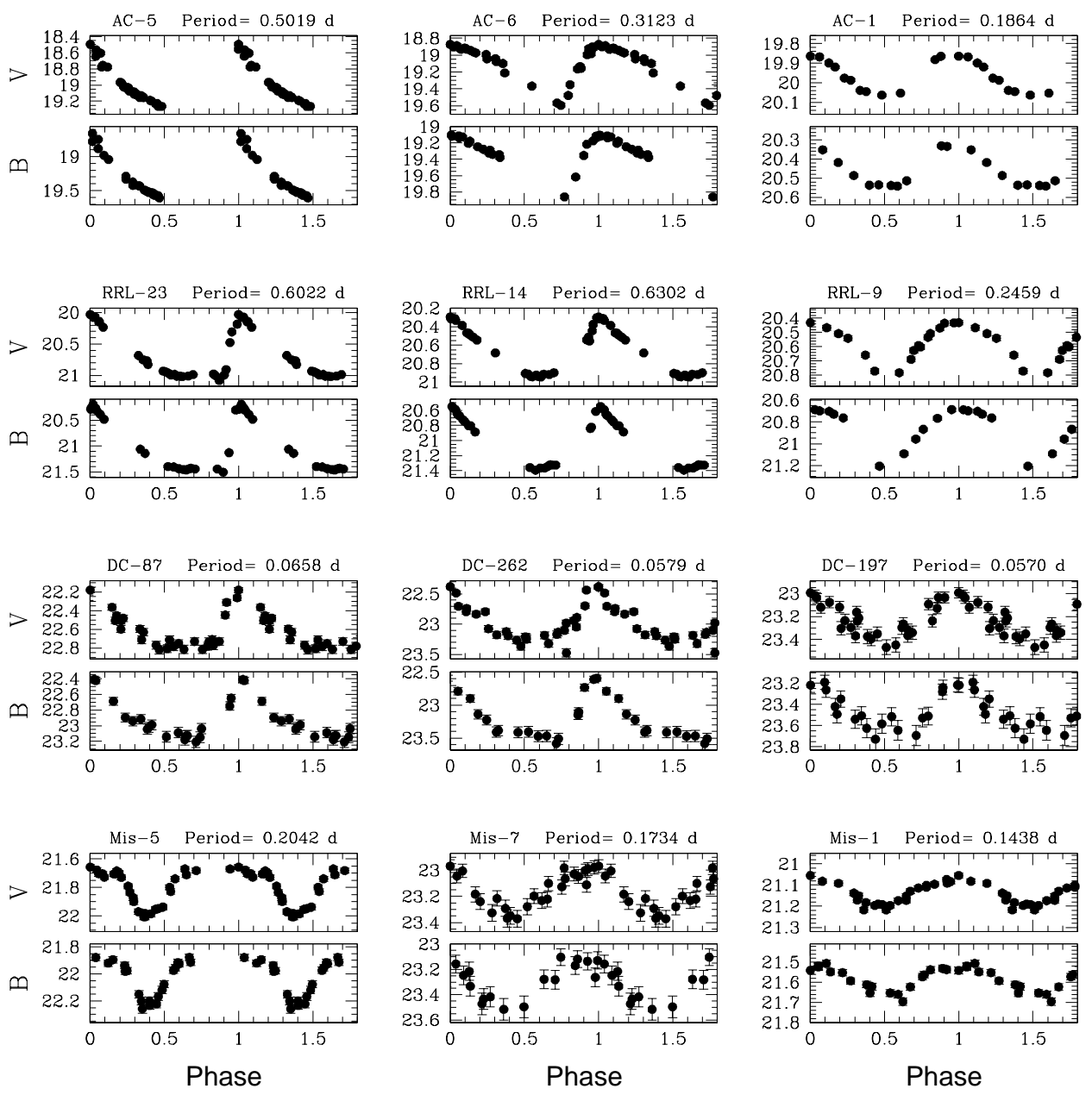

Fig. 7.- Examples of light curves. From top to bottom, each row presents examples of anomalous Cepheids, RR Lyrae stars, DC and stars in the miscellaneous group. 


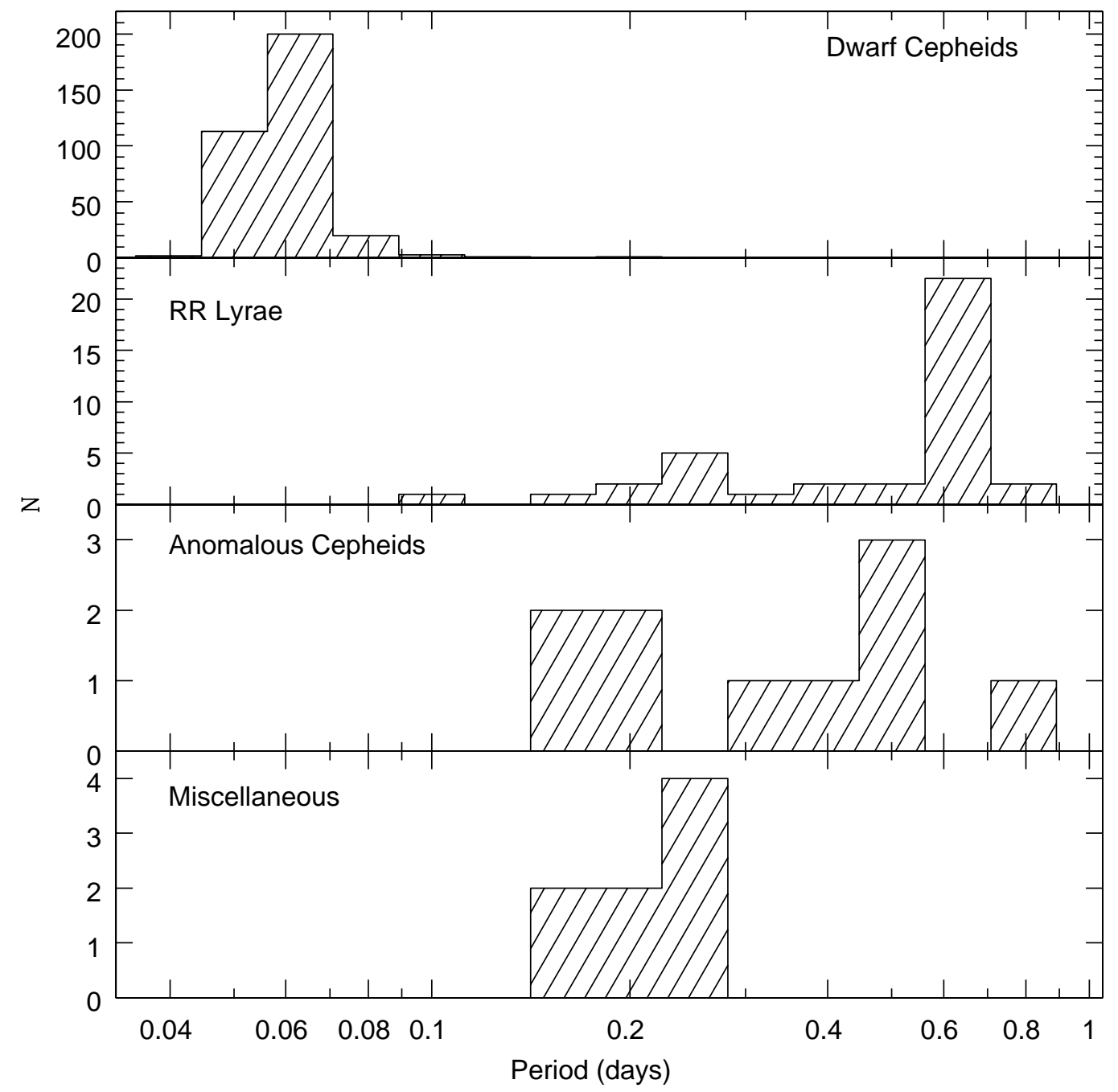

Fig. 8.- Period distribution of stars in the four groups of variables identified in this work. 
They found 75 RR Lyrae stars. Even with our non-ideal sampling for this type of stars, we recovered 30 of them. The ID number in D03, together with their type classification and period, is included in Table 2. The 20 type RRab in common with the sample in D03 agree in their periods within $0.01 \mathrm{~d}$. We also recovered the correct dominant period for 1 type $\mathrm{d}$ star. However, we did not attempt to find double mode pulsators, hence, type d stars in D03 were classified here as either type ab or c. We recovered aliases for all the type c, and the two remaining type d stars in common. Type c stars are most susceptible to aliasing problems since the $\pm 1 \mathrm{~d}$ alias correspond to periods within the expected range for these type of stars (e.g. Vivas et al. 2004). Three stars (RRL-6, RRL-10 and RRL-29), which were classified by us as RRc, were labelled as RRab in D03. Most likely the disagreement is due to our poor phase coverage for some of these star. Since D03 had lightcurves with a significantly higher number of epochs, we recommend the use of their periods and classification for the stars in common. Figure 9 shows a zoomed version of the CMD in the region of the bright variables. All of the RR Lyrae stars concentrate in a narrow range of magnitudes. The mean magnitude of the 38 stars detected in this work is $V=20.71$, with a standard deviation of only 0.12 mags. This value agrees well with the mean found by D03 for their whole RR Lyrae sample, $V=20.68 \pm 0.06$.

By looking at the spatial distribution of the RR Lyrae stars in our sample (Figure 10), it is clear that the detection of this type of stars was only possible where we had overlap among fields (see Figure 1), and hence we had a larger number of epochs. Stars in the D03 catalog that we did not recover were located mostly outside of the overlap regions and hence, they did not have enough epochs and phase coverage in our survey to be recognized as RR Lyrae stars. We recall that in the regions with no overlap observations were limited to several exposures within a period of $\sim 4$ hours, with only 1 or 2 additional exposure on a different night. In most circumstances, this will not sample a significant part of the light curve of an RR Lyrae star, most especially, for the ones with longer period.

Eight new RR Lyrae stars in Carina are reported in this work; they are marked with open circles in Figures 9 and 10. Six of them are located outside the field studied by D03, and far from the center of the galaxy.

\subsection{Anomalous Cepheids}

We found 10 periodic variables brighter than the horizontal branch (see Figures 6 and 9) which we identify as anomalous Cepheids in Carina. Six of these had been previously classi-

fied as such by D03. Out of these 6 stars, we recovered the period within $<4 \%$ for three of them (AC-2, AC-3 and AC-5); for two of them we recovered alias periods (AC-6 and AC-7); 


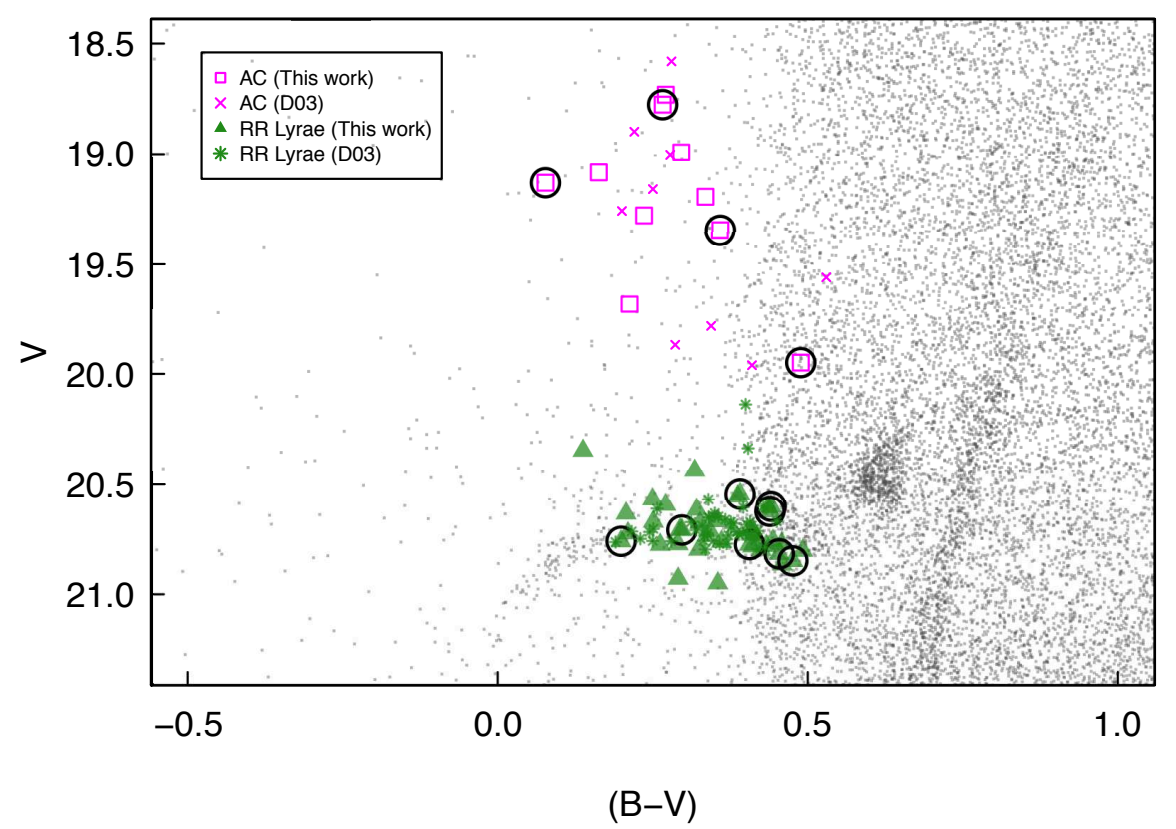

Fig. 9.- Enlargement of the CMD in the region o the bright variables. RR Lyrae stars and anomalous Cepheids identified in this work are indicated by the triangles and open squares respectively. New discoveries are enclosed by open circles. Stars in D03 not recovered in this work are shown with asterisks ans $\times$ symbols for RR Lyrae stars and anomalous Cepheids respectively. 

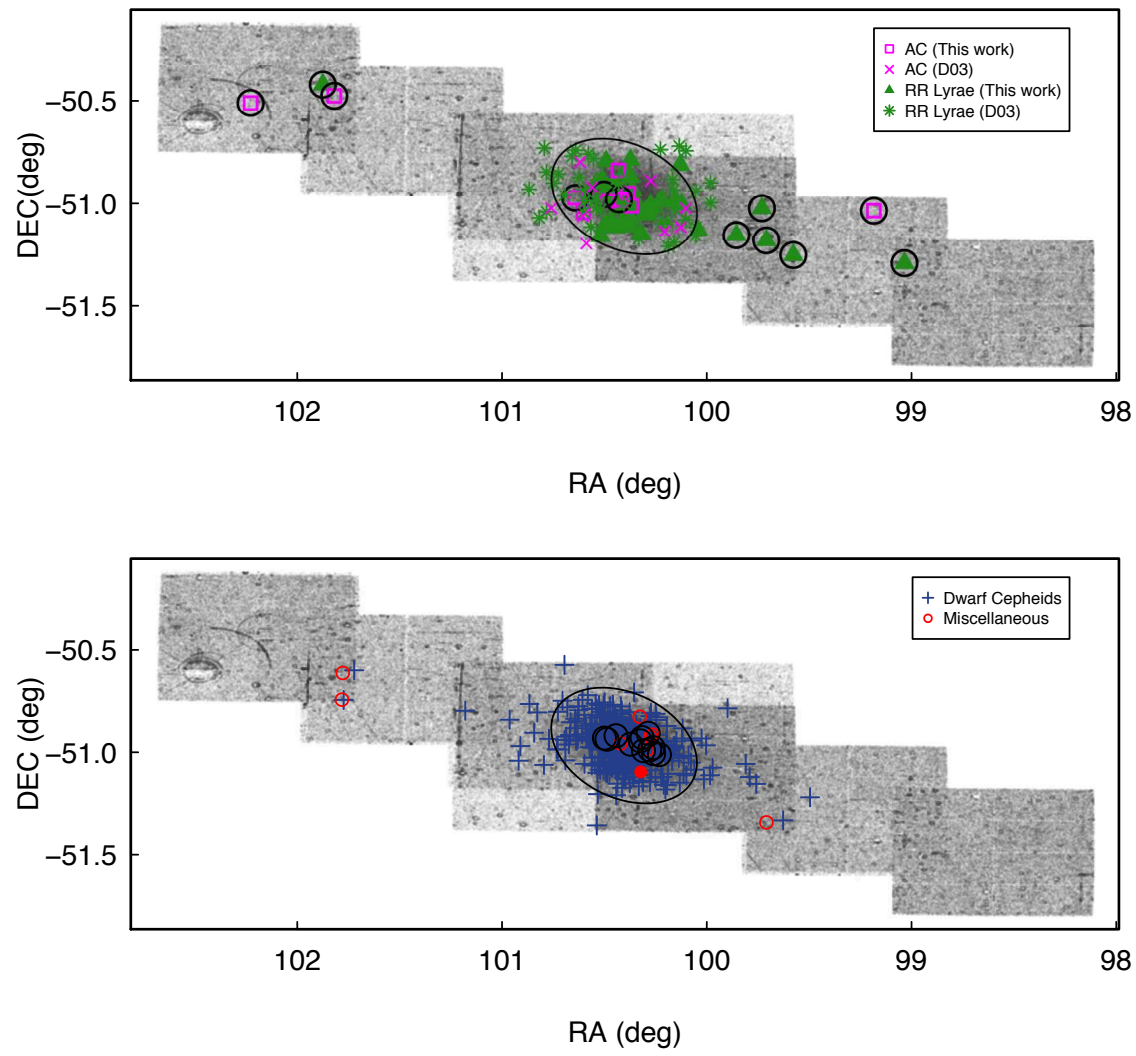

Fig. 10.- Distribution in the sky of the periodic variables detected in this work. The top panel shows the bright variables (RR Lyrae stars and anomalous Cepheids) while the bottom panel shows the faint ones (DCs and miscellanous stars). Symbols are the same as in Figure 9 and 11. While open black circles in the top diagram enclose the new RR Lyrae stars and anomalous Cepheids discovered in this work, in the lower diagram the same symbol is used to mark the previously known DC from Mateo et al. (1998). In both plots the ellipse represents the tidal radius (22'.54), ellipticity (0.32) and position angle $\left(64^{\circ}\right)$ of Carina, according to Walcher et al. (2003). 
finally, for one star (AC-4) the period reported by D03, $1.16 \mathrm{~d}$, was outside the range we studied in this work. However, we found a period for that star of $0.55 \mathrm{~d}$, which produces a nice and smooth light curve. We notice that one period is close to the harmonic of the other. As with the RR Lyrae stars, since the light curves of D03 contains more epochs than ours, their periods are likely the correct ones for these longer period variables. Examples of three light curves of anomalous Cepheids are shown in the top row of Figure 7.

We report finding four new anomalous Cepheids, three of them located outside the region studied by D03 and some of them up to $\sim 1^{\circ}$ from the center of Carina (Figure 10). Table 3 contains the data for all the anomalous Cepheids found in this work. The table includes the ID and period determined by D03. With these 4 new stars the total number of known anomalous Cepheids in Carina is 10.

\subsection{Dwarf Cepheids}

This is by far the most numerous kind of variables we found in our study. This partly reflects our observing strategy which was designed specifically to maximize the detection and recovery of these types of short period variables, but also the sheer high frequency of DCs in Carina. With the identification of 340 DC stars, Carina is one of the stellar system with the largest known number of this type of stars, only surpassed in total number by the LMC (Garg et al. 2010). The only other external system to the Milky Way known to contain a large number of DCs is the Fornax dSph galaxy (85 DCs, Poretti et al. 2008).

Mateo et al. (1998) looked for DCs in three small fields in Carina $\left(3^{\prime} .9 \times 33^{\prime} 9\right.$ each), finding 20 of these variables. We confirmed 12 of them in this work for which we recovered periods within $10^{-3} \mathrm{~d}$ (this difference includes that we may have recovered the 1-day alias for some stars). The stars in common with the sample in Mateo et al. (1998) are V4, V5, V9, V10, V12, V13, V14, V15, V16, V17, V18 and V20 (these IDs correspond to the ones assigned by Mateo et al). Out of the 8 stars which were not recognized as dwarf cepheids, 2 of them (V6 and V8) show variability in our lightcurves but no period within the searched range was found. The variability of the remaining 6 stars is more uncertain in our data. Some stars (V3, V7 and V19) present either variability at the level $P\left(\chi^{2}\right)<0.1$ (instead of $P\left(\chi^{2}\right)<0.01$ ), sometimes in the $\mathrm{V}$ band only, or no variability at all (in the case of V1, V2 and $\mathrm{V} 11)$.

Figure 11 shows a zoom of the CMD in the region of the faint variables. The location of the previously known DC stars from Mateo et al. (1998) are indicated with thick open circles. The 340 DC stars are strongly concentrated in color, exhibiting a mean sample $(\langle B\rangle-\langle V\rangle)$ 
of $0.26 \pm 0.06 \mathrm{mag}(1 \sigma)$. On the other hand, there is a relatively larger spread in brightness. Although most of the stars clump around magnitude $V \sim 23.1$, there is a large tail toward brighter magnitudes, with a secondary peak at $V=22.6$, and maybe a third concentration at $V=22.35$. We will see below that these different peaks may be associated with different pulsation modes of the DC stars.

All of the identified DC stars have relatively large amplitudes. The distribution of amplitudes in the $\mathrm{B}$ and $\mathrm{V}$ bands are shown in Figure 12. In the B band, stars show variations between 0.2 and 1.6 magnitudes, with a mean value of 0.6 magnitudes. The $\mathrm{V}$-band amplitudes range from 0.2 to 1.1 with a mean of 0.5 magnitudes. However, these mean values may be biased toward larger mean amplitudes since it is clear from Figure 12 (bottom) that we may be missing lower amplitude variables $(\lesssim 0.4)$ at the faintest magnitudes. Figure $13 \mathrm{a}$ shows the relationships between the amplitudes in the B and V bands. Most of the stars lie below the 1:1 line indicating that, like other pulsating stars, amplitudes in the B band are larger than in $\mathrm{V}$. The mean ratio between the amplitudes, Amp B/Amp V, is 1.2.

A period-amplitude diagram (Figure 13b) shows no correlation at all between these two properties of the light curve. It is well known that such a relationship exists for RR Lyrae stars (eg Smith 1995) with the lower amplitude variables having the longest periods. However this seems not to be the case for DC variables.

Table 4 contains the properties of all the DC stars identified in this work. The table includes the suspected alternative periods (aliases), and the ID and periods determined by Mateo et al. (1998). Stars within the locus of the fundamental mode pulsators (see $\S 6$ ) are identified with an "F" in column 13.

\subsection{An Eclipsing Binary and other periodic variables}

Out of the group of miscellaneous stars, Mis-5, shows a light curve typical of eclipsing systems (bottom left panel in Figure 7). We measured a period of $0.204 \mathrm{~d}$ for that star. The location of this star in the CMD is shown in Figure 11. It is separated from the main bulk of DC stars previously discussed.

The remaining 8 periodic variables in this group do not seem to fit in any of the groups discussed above. They are marked with small open circles in Figure 11. The periods of this group of stars range 0.14 to 0.25 days, which are higher than what we found for the majority

of DC stars (see Figure 8). However, they do not occupy a clear region in the CMD. They may be eclipsing systems with sinusoidal light curves. Properties of these stars are listed in Table 5 . 


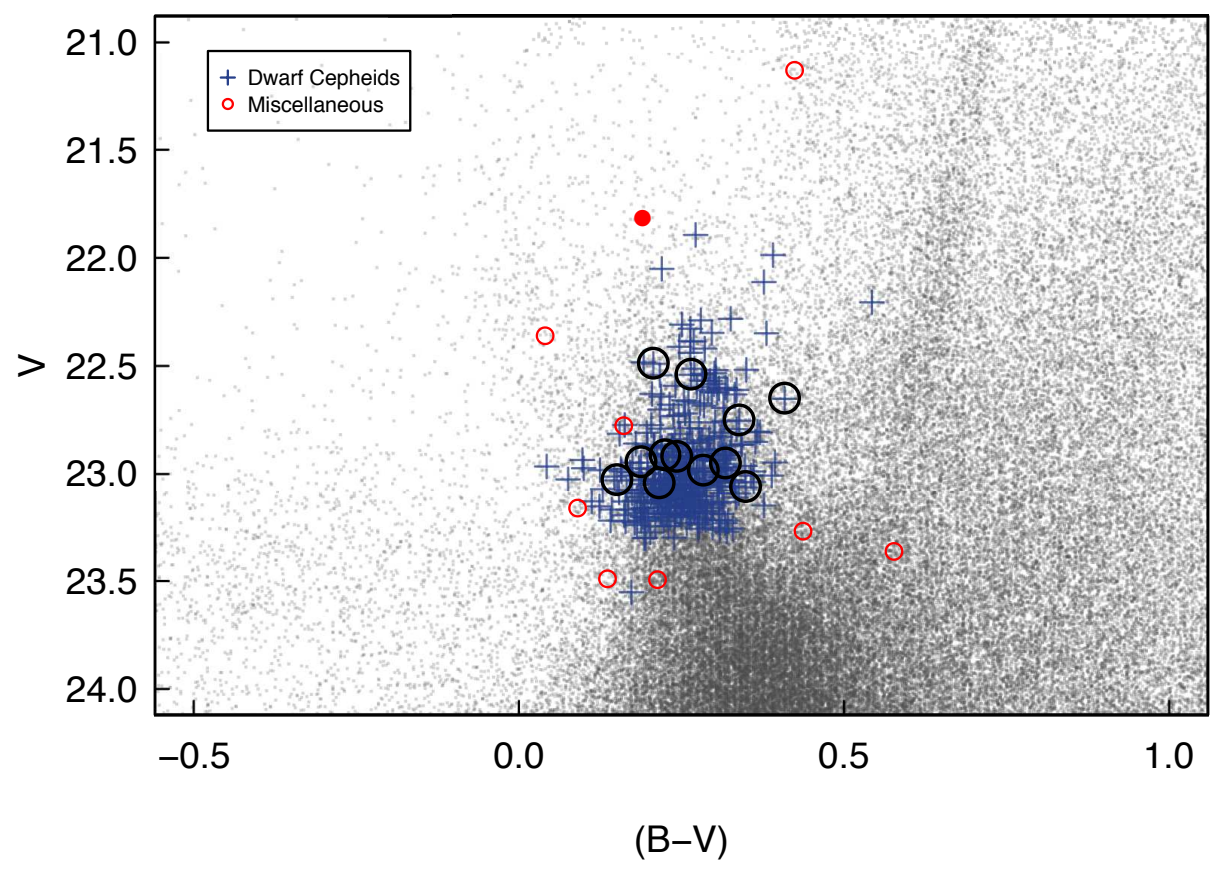

Fig. 11. - Enlargement of the CMD in the region o the faint variables. DC stars are indicated by the + symbols. The clear example of an eclipsing binary is the solid circle, while the rest of the stars in the miscellaneous group are shown with small open circles. Previously known DC stars in Carina (Mateo et al. 1998) are enclosed by thick open circles. 

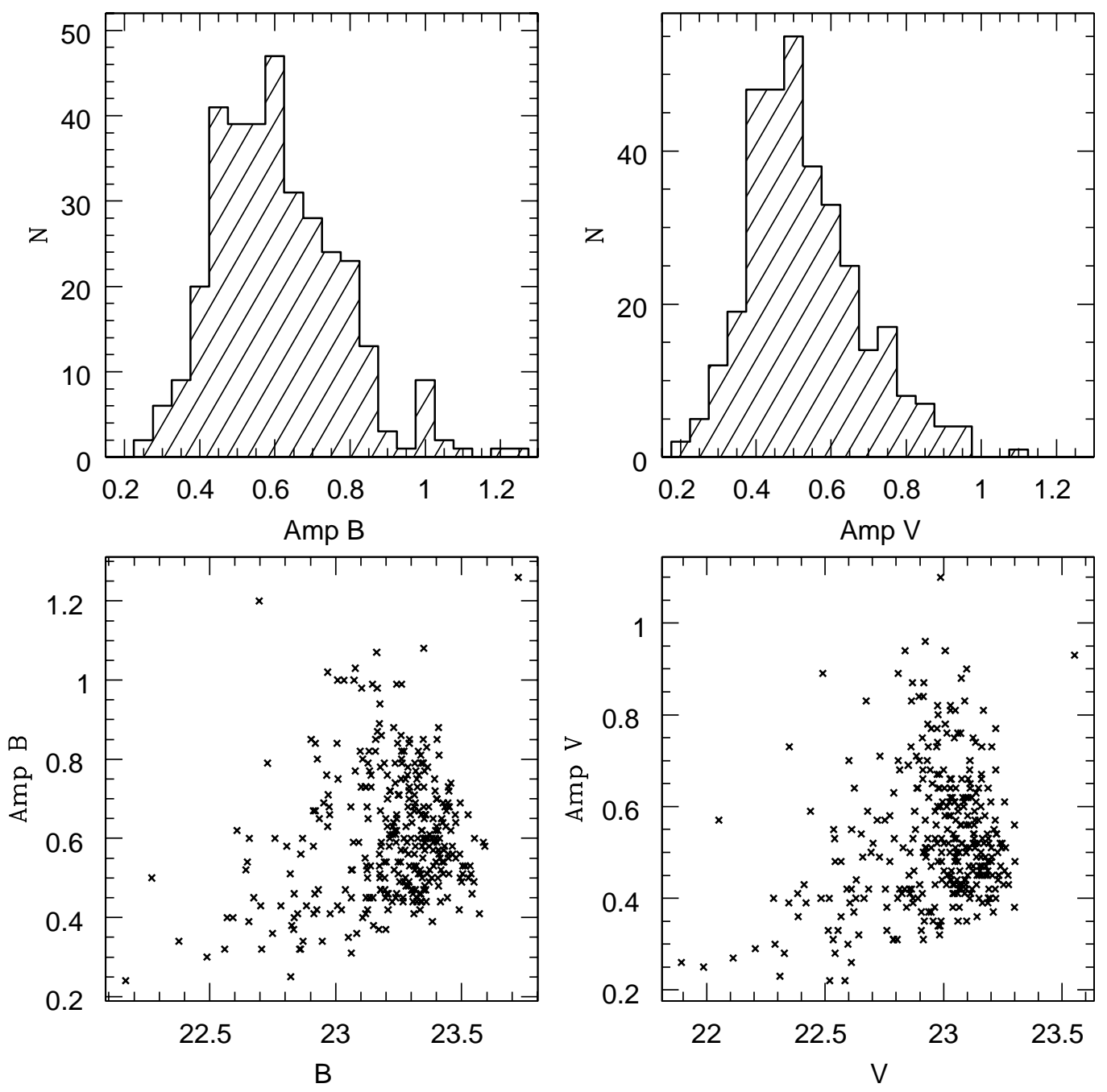

Fig. 12.- (Top): Distribution of the amplitudes of the DC stars in the B and V band. (Bottom): Amplitudes in B and V as a function of the mean magnitude. 

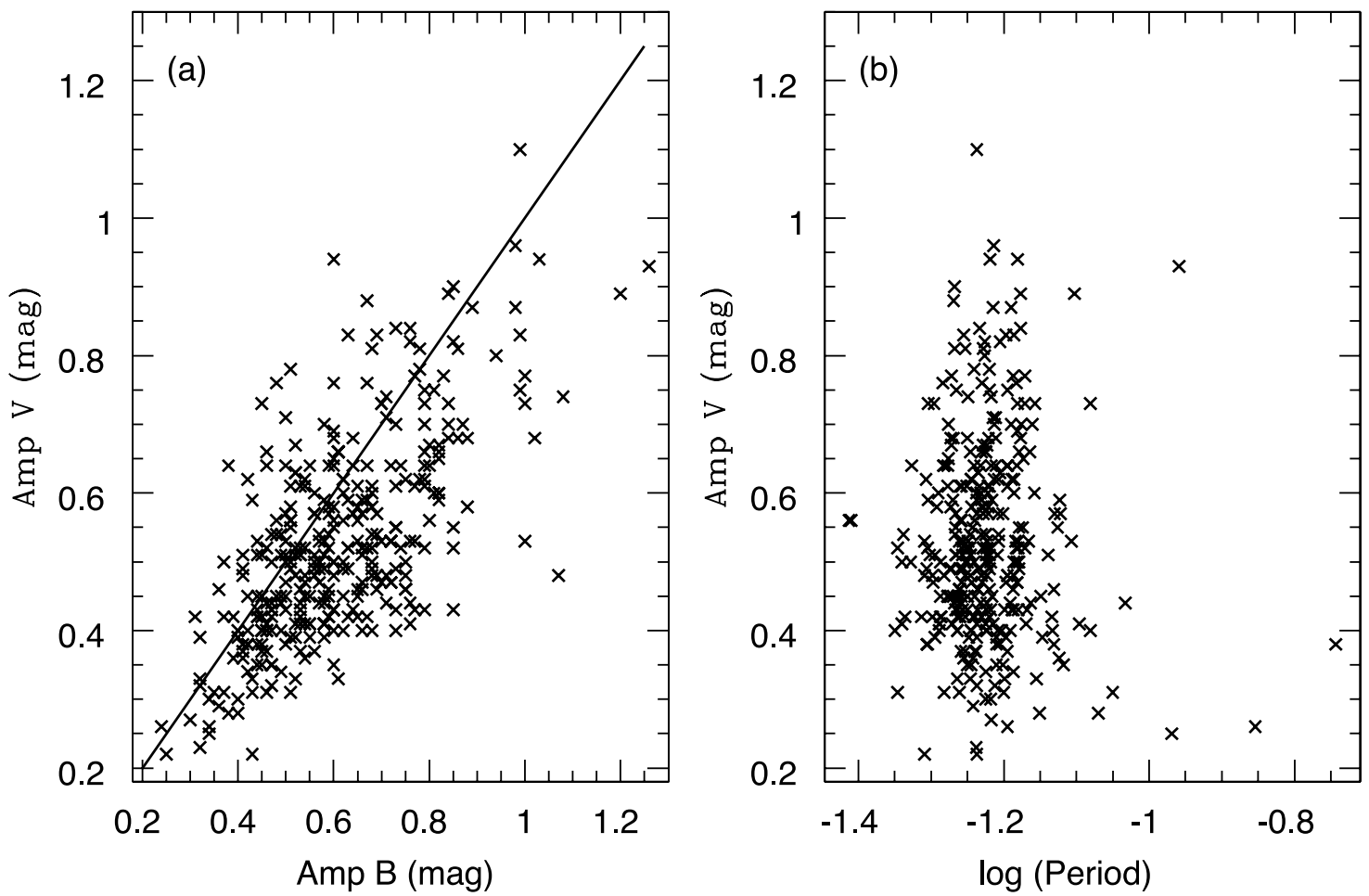

Fig. 13.- (a) Relationship between the amplitudes of DC stars in the B and V band. The solid line is a 1:1 relationship. (b) Relationship between the amplitude in the $\mathrm{V}$ band and the logarithm of the period.

\section{Extinction}

Since the sky coverage of this survey is large, we determined reddenings toward each of the individual DC stars using the dust maps of Schlegel et al. (1998) instead of using a mean extinction for the whole galaxy. The mean galactic latitude of Carina is $b \sim-22.2$, which is low enough to expect variations in extinction across the survey area. Figure 14 shows a map of the $A_{V}$ extinction derived from the dust maps of Schlegel et al. (1998) ${ }^{1}$. It is clear from that map that there is a significant extinction gradient along the survey area, with the SW extreme of the survey having about twice the redening than the other extreme. In the central region of the galaxy the color excess, $\mathrm{E}(\mathrm{B}-\mathrm{V})$, spreads over $\sim 0.01$ mags, which translates in differences in $A_{V}$ of the order of $0.03 \mathrm{mag}$. The mean value of the color excess of the DC stars is $\langle E(B-V)\rangle=0.063$ mags with a standard deviation of 0.004 mags. Using $A_{V}=3.240 \times E(B-V)$ and $A_{B}=4.325 \times E(B-V)$ (Schlegel et al. 1998), the mean

\footnotetext{
${ }^{1}$ We used the tools available at the NASA/ IPAC Infrared Science Archive (http://irsa.ipac.caltech.edu/applications/DUST/)
} 


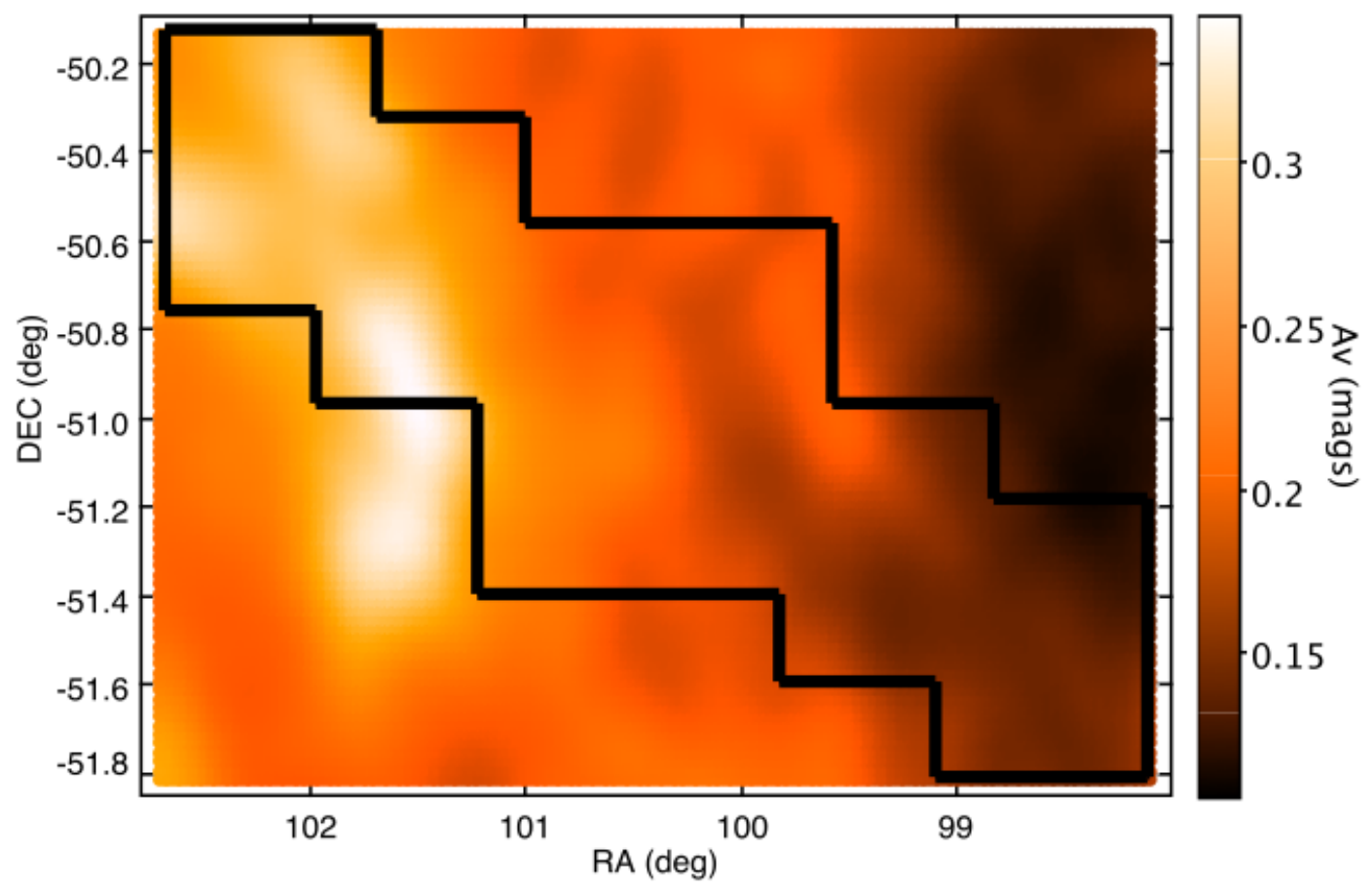

Fig. 14.- Extinction map from Schlegel et al. (1998) over the region of the survey. The solid black lines enclose the observed region.

extinctions are $\left\langle A_{V}\right\rangle=0.20$ mags and $\left\langle A_{B}\right\rangle=0.27$ mags.

\section{Period-Luminosity Relationship}

DC stars can be used as standard candles since they exhibit a period-luminosity (P-L) relationship. A difficulty is that there are different relationships for stars pulsating in the fundamental $(\mathrm{F})$ or first overtone (FO) modes, with the latter being brighter for a given period. Thus, identification of the pulsation mode is is crucial to assigning the proper P-L locus to a given star. Contrary to the RR Lyrae stars for which it is relatively easy to identify the pulsation mode based in the periods and shape of the lightcurves (type ab and c), in DC stars the separation is more complex. Not only the periods of both types of pulsators overlap but also, a "sawtooth" or sinusoidal light curves does not necessarily correspond with fundamental and first overtone pulsators (Mateo et al. 1998; Poretti et al. 2008; Cohen \& Sarajedini 2012). Location in the CMD also does not help to separate stars by mode (Cohen \& Sarajedini 2012). 


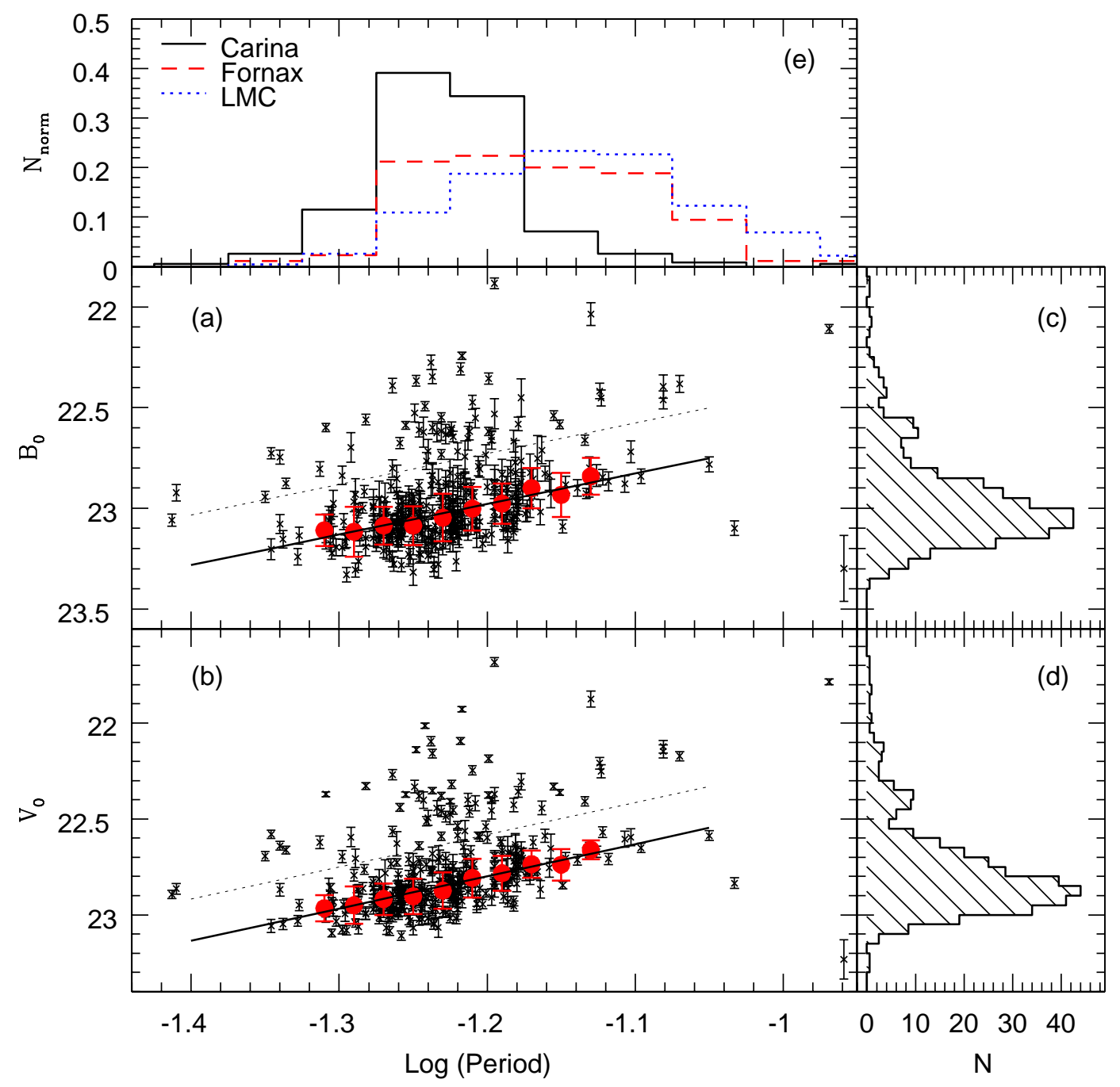

Fig. 15.- (a-b) Relationship between the extinction corrected magnitudes versus de logarithm of the period of the 340 DCs found in this work $(\times$ symbols $)$. The stars pulsating in the fundamental mode are mostly located below the dotted line. Large solid points are averages of the brightness of the fundamental mode pulsators in bins of 0.02 in $\log (P)$, and the error bars are the $1 \sigma$ spread in each bin. Only bins with 5 or more stars were considered. The solid line is the best fit through the red points. (c-d) Distribution of the extinction corrected brightness in the B and V bands. (e) Distribution of the periods of DCs in Carina, Fornax and LMC. 
Plots of the extinction corrected $B_{0}$ and $V_{0}$ magnitudes of the DC stars in Carina versus the logarithm of their periods (Figures 15(a) and 15(b)) show several interesting things. First, it is clear that the DC stars in Carina show a P-L relationship since fainter stars tend to have shorter periods. Second, the distribution of periods of the DC stars in Carina (panel e in Figure 15) has significant differences with the ones in the Fornax dwarf spheroidal galaxy and in the LMC; we defer a more detailed discussion about this issue for next section. Finally, the distribution of stars in this diagram is not uniform in magnitude. Two groups or sequences of stars are separated by a gap containing very few stars. The fainter group is the most numerous containing about $80 \%$ of the whole sample. Then, a brighter group seems to run parallel to the faint one but $\sim 0.35$ mags brighter. There is a hint of a third sequence at even brighter magnitudes. This uneven distribution can also be seen in the histograms of magnitudes in the right panels of Figure 15 (panels c and d), in which two (maybe three) peaks are observed, corresponding approximately with the sequences just described. The behaviour is approximately the same in both the $\mathrm{B}$ and the $\mathrm{V}$ bands.

To make a more quantitative assessment of the distribution of the stars in this diagram, we drew an arbitrary line through the gap and fitted a straight line to the most numerous group, that is, all the stars below the line. Then, we calculated the projected distance of each star to the best fitted line (Figure 16). A histogram of the distribution of the projected distances in the $\mathrm{B}$ and $\mathrm{V}$ band clearly shows that the uneven distribution is well modeled by three Gaussian curves corresponding to the sequences described above. The smallest Gaussian corresponding to the brightest sequence is better seen in the B data, and just barely in V. The minimum point between the two main Gaussian curves in Figure 16 allowed us to better define the separating line between the two strongest sequences of stars in the diagram. After a few iterations in this process we found the best separation of the sequences, which is shown as a dotted line in Figure 15.

With only 20 DC stars in Carina available at that time, Mateo et al. (1998) was already able to distinguish the two strongest sequences observed in Figure 15, and recognized the faintest one as stars pulsating in the $\mathrm{F}$ mode, while the brightest sequence corresponded to FO pulsators. This was made by realizing that the PL relationships for these two types of pulsators, as given by Nemec et al. (1994), fitted very well their data and implied a distance modulus for Carina of $\mu_{o}=20.06$, in good agreement with other measurements in the literature. Assuming this distance, the Nemec et al. (1994)'s PL relationships (which include a metallicity term for which we assumed, as Mateo et al. did, $[\mathrm{Fe} / \mathrm{H}]=-2.0$ ) pass over the two sequences observed in our data as well, confirming that the bulk of the DC stars in Carina should be stars that are pulsating in the F mode, and the parallel brighter sequence corresponds to stars pulsating in the FO. The brightest stars in the sample may be pulsating in even higher overtones. 


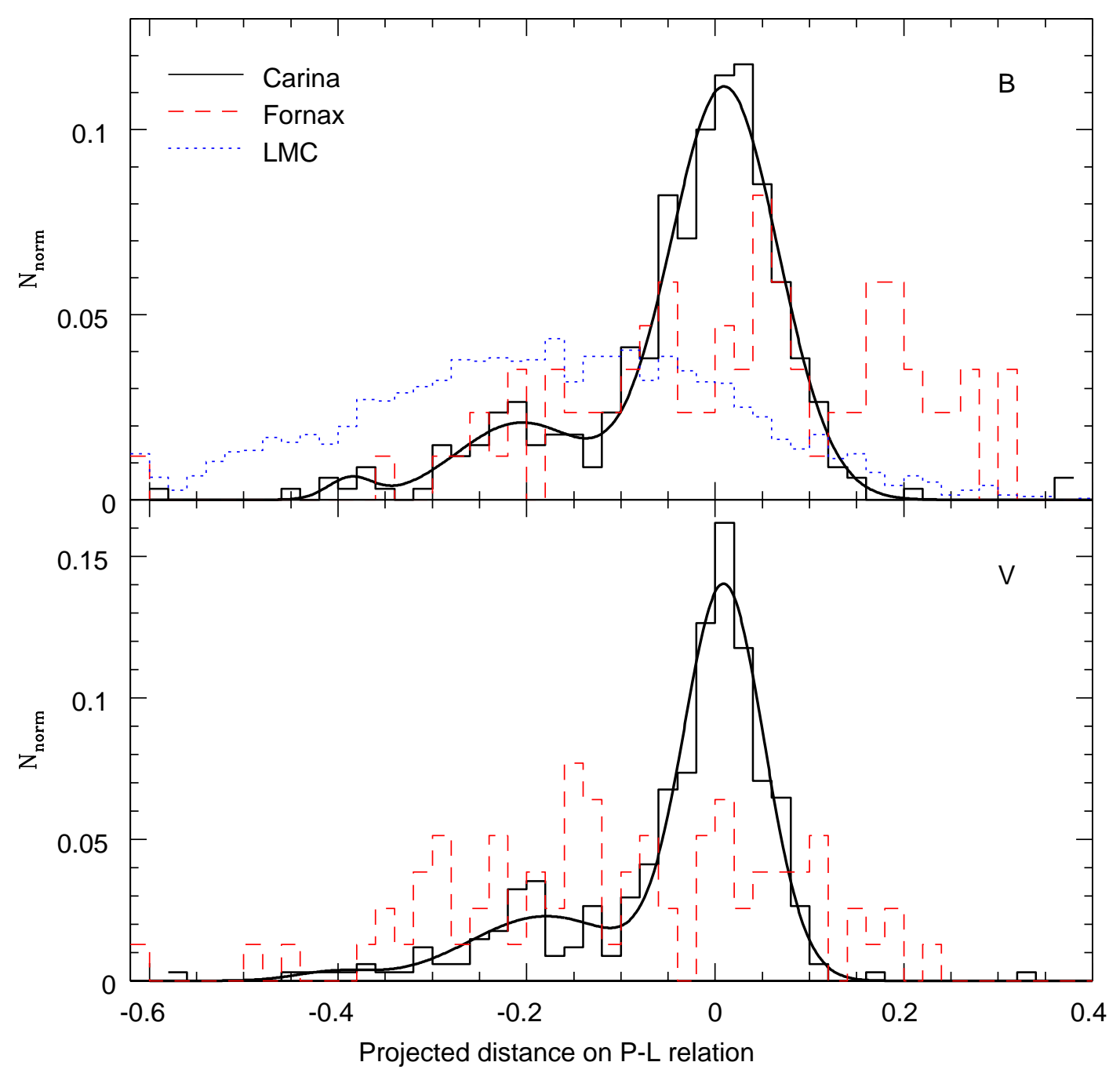

Fig. 16. - Histogram of the projected distance of all DC stars on the P-L relationship determined for Carina (equations 4 and 5). The distribution of the projected distances in Carina is well modelled by three Gaussian curves (thick solid line), which we interpreted as groups of DC stars pulsating in different modes. For comparison, the distributions for Fornax and the LMC are also shown as dashed and dotted histograms. A distance modulus of 20.72 and 18.47 was assumed for Fornax and the LMC respectively in order to calculate the relative distance on the P-L relationship of Carina. All histogram have been normalized by the total number of DCs in each galaxy. 
We thus selected F pulsators as the stars below the arbitrary line shown in Figure 15. Because we see such a clear distinction among the DCs in Carina, we can readily investigate the pulsational properties of the DCs as a function of mode. For example, upon examination of some of the light curves of stars pulsating in different modes, we arrived at the same conclusion as previous authors (Mateo et al. 1998; Poretti et al. 2008) that the shape of the light curve is not a indication of the pulsation mode. The third row of lightcurves in Figure 7 shows three examples of DC stars in our sample. The middle and right column (DC-262 and DC-197) show two stars in the F pulsation locus. One of them has a clear sawtooth-type lightcurve while the other is almost sinusoidal. The star in the left column (DC-87) is a FO pulsator according to its location in the PL diagram. It has a sawtooth lightcurve. There are more examples of similar cases in our Carina dataset.

To determine the P-L relationship for the Carina DCs, we calculated the average brightness of $\mathrm{F}$ pulsators in 0.02 dex bins in $\log (P)$ that contained five or more stars; these binned averages are shown as large dots in Figure 15. This method gives equal weight along the full period range while minimizing the effects of outliers. We then use a least squares method to fit a straight line through those points (solid lines). We found the following relationships:

$$
\begin{aligned}
& B_{0}=21.16-1.520 \log (P) \\
& V_{0}=20.78-1.682 \log (P)
\end{aligned}
$$

As mentioned before, the P-L relationships given by Nemec et al. (1994) fitted well the earlier data of DC stars in Carina. However, the best fit to these new Carina data has a shallower slope than the P-L relationship in Nemec et al. (1994), which for the F mode pulsators in the $\mathrm{V}$ band is:

$$
M_{V}=-2.56( \pm 0.54) \log (P)+0.32[\mathrm{Fe} / \mathrm{H}]+0.36 ; \quad(\text { Nemec et al 1994) }
$$

In recent years there have been several efforts in establishing precise P-L relationships for DC stars which have even steeper slopes than Nemec et al. (1994). If the metallicity of the population is known, McNamara (2011) has proposed a metallicity-dependent P-L relationship for DC stars:

$$
\begin{array}{ll}
M_{V}=-2.90( \pm 0.05) \log (P)-0.19[\mathrm{Fe} / \mathrm{H}]-1.27 ; & \text { if }[\mathrm{Fe} / \mathrm{H}]>-2.0 \\
M_{V}=-2.90( \pm 0.05) \log (P)-0.89 ; & \text { if }[\mathrm{Fe} / \mathrm{H}] \leqslant-2.0
\end{array}
$$


In practice, for extragalactic systems it is typically not straightforward to define a single metallicity for purposes of defining the P-L relation for a DC population (and especially since both SX Phe and $\delta$ Scuti stars may coexist in the same place). Poretti et al. (2008) and Cohen \& Sarajedini (2012) have constructed P-L relationships by joining datasets from galactic globular cluster and some extragalactic systems (namely, Fornax, Carina and the LMC; the latter included only in Cohen \& Sarajedini (2012)'s relationship). The resulting P-L equations are:

$$
\begin{gathered}
M_{V}=-3.65( \pm 0.07) \log (P)-1.83 ; \quad(\text { Poretti et al 2008) } \\
M_{V}=-3.389( \pm 0.09) \log (P)-1.640 ; \quad(\text { Cohen \& Sarajedini 2012) }
\end{gathered}
$$

We applied all those P-L relationships to our data to calculate the distance modulus to Carina using its 268 F-mode DC pulsators by determining the best-fit for the fixed slopes of relations 6 to 9 . From the intercept of the best-fit lines in combination with the equations above we derived a de-reddened distance modulus for Carina. Table 6 summarizes the results. For the relationships that have a dependency on metallicity, we used both $[\mathrm{Fe} / \mathrm{H}]=-2.0$ and -1.7 , although the latter may be more representative of the intermediate/old population of Carina (Monelli et al. 2003). For $[\mathrm{Fe} / \mathrm{H}]=-1.7$, the average of the distance modulus given by equations 6 to 9 is $\mu_{0}=20.17 \pm 0.10$ mags, which is in very good agreement with the estimate from RR Lyrae stars by D03 $\left(\mu_{0}^{R R}=20.12 \pm 0.12\right)$.

\section{Comparison with the Fornax Dwarf Spheroidal Galaxy and the LMC}

Numerous DC stars have also been found in the Fornax dwarf spheroidal galaxy by Poretti et al. (2008). These stars in Fornax are referred as SX Phe by Poretti et al. (2008) since they expect most of the stars in this galaxy to be metal-poor. Like Carina, Fornax contains a complex stellar population with a prominent intermediate age population (Coleman \& de Jong 2008) and a well populated main sequence at the intersection with the instability strip. Garg et al. (2010) identified 2,323 DC stars (referred in this case as HADS) in the LMC using the SuperMACHO dataset. The number of DC stars in Carina, Fornax and the LMC are large enough to allow detailed comparisons on their properties. Three SX Phe have been reported in the globular cluster M54 located at the center of the Sagittarius dSph galaxy (Sollima et al. 2010) but they are too few to make any firm comparison of their properties. To our knowledge, these are the only extragalactic DC systems known. 


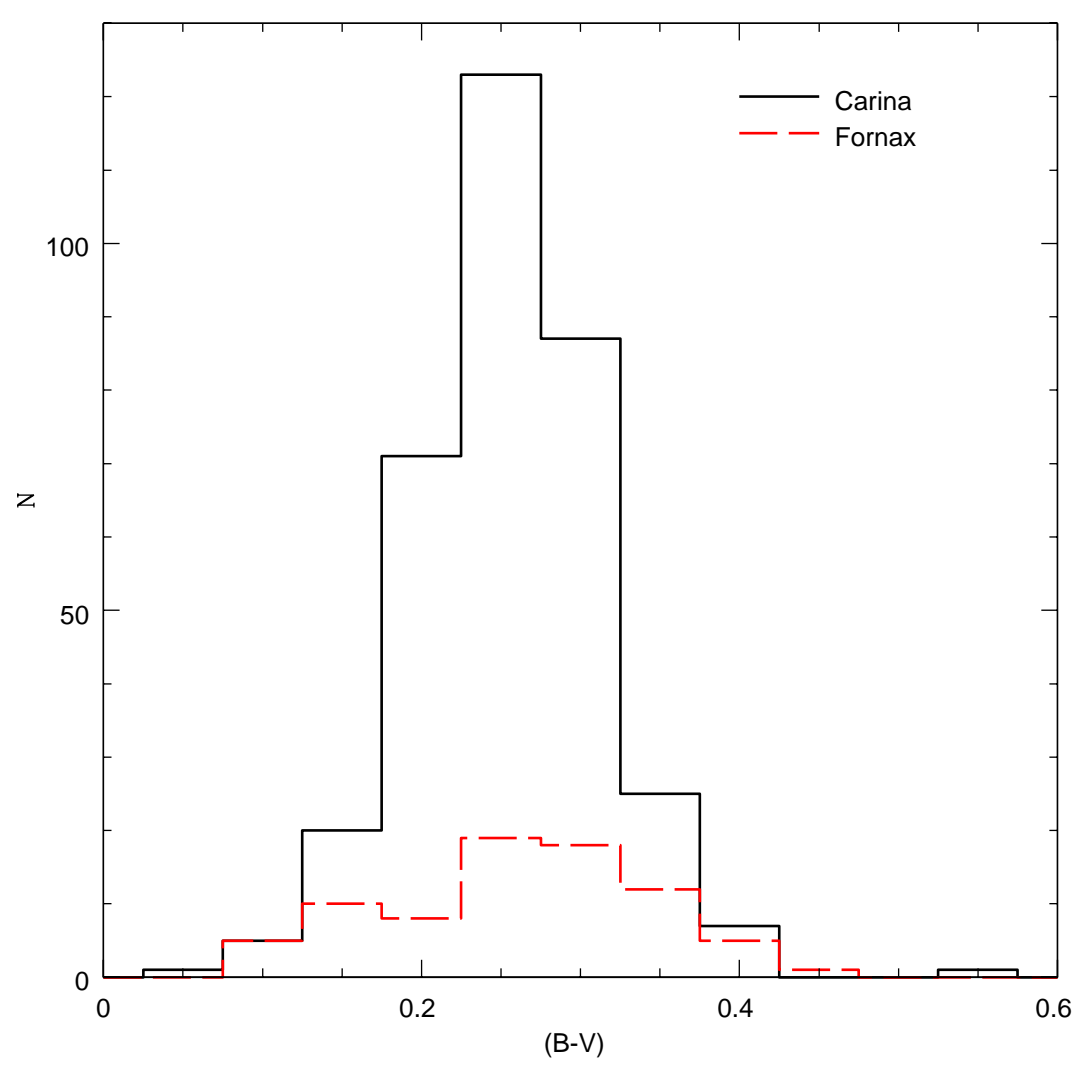

Fig. 17. - Color distributions of DC stars in the Carina (solid histogram) and Fornax (dashed histogram) dwarf spheroidal galaxies.

Based on the color distribution of the DC stars (Figure 17) we find that the width of the instability strip is remarkably similar in both Carina and Fornax. In both cases, most of the DC stars are confined to the color interval $0.10<B-V<0.40$. Poretti et al. (2008) make the case, however, that this width may be larger than what is observed in the Milky Way. We could not compare with the color distribution in the LMC since the only color provided by Garg et al. (2010) is $B-I$.

The DC stars in the three galaxies have very short periods $(<0.1 \mathrm{~d})$. However, Figure 15 (panel e) shows that the period distribution of the Carina DCs is the shortest of the three. There seems to be a sequence among the three galaxies with the distribution of periods shifted toward longer periods when going from Carina, to Fornax and to the LMC. The median value of the periods in Carina is 0.059 days, while in Fornax is 0.067 days and in the LMC is 0.073 days. We notice that the mean metallicity in these galaxies has the same sequence with Carina being the most metal-poor of the three. 
Aliasing should not be responsible for the unequal period distributions since the differences between the true periods and their most common aliases are very small, much smaller than the bin size of the histograms in Figure 15 for stars with periods $<0.1 \mathrm{~d}$. On the other hand, it is possible that incompleteness may play a role, at least in part, for the differences observed in the distributions. Stars with the shortest periods are also the faintest ones (they follow a P-L relationship). In the case of Fornax (as in Carina) the limits on the minimum amplitude for the detection of variables increases with magnitude (see our Figure 12 and Figure 2 in Poretti et al. (2008)). Hence, stars with small amplitudes are likely missing at the faintest magnitudes. The minimum amplitude observed in DC variables in these two galaxies varies as a function of magnitude approximately in the same way within the range of magnitudes of the DC stars. Thus, incompleteness should affect both galaxies similarly, while for the LMC incompleteness may not be significant since the minimum amplitude detected remains constant as a function of magnitude, at least in the range of interest of these stars.

Both Fornax and the LMC have a larger relative number of stars with periods $>0.075$ $\mathrm{d}$, which are rare in Carina. Similarly, but not as striking, the LMC has a relatively large number of stars with periods $>0.095 \mathrm{~d}$, which are rare in Fornax. Kolmogorov-Smirnov tests reject the hypothesis that the samples are drawn from the same parent population at the $99.9 \%$ confidence level. The longer periods among the DC stars in the LMC can be understood if this galaxy contains mostly $\delta$ Scuti stars (specifically, HADS), which is indeed the identification given by Garg et al. (2010) to those stars. HADS stars tend to have longer periods than SX Phe, although significant overlap in the period distributions of both types exists (McNamara 2011).

Another remarkable difference in the population of DC stars among these three galaxies has to do with the distribution of stars in the P-L diagram. As mentioned in section 6, the stars pulsating in the F mode in Carina are clearly separated in the diagram by a gap containing almost no stars. However, that is not the case for Fornax and the LMC. In Figure 18 we show the P-L diagrams for the three galaxies. Fornax and the LMC show a wider distribution (in both periods and brightness) with no obvious gaps or distinct sequences apparent. This is more clearly seen in Figure 16 in which we plotted the projected distance of the stars in the three systems on the P-L relationship determined for Carina (equations 4 and 5), after taking into account the difference in distance modulus of those galaxies with respect to the value of 20.17 we found in this work for Carina (we assumed a distance modulus of 20.72 for Fornax (Rizzi et al. 2007) and 18.47 for the LMC (Cohen \& Sarajedini 2012)). As we explained in the previous section, in Carina this distribution is well modeled with three Gaussian curves which we interpreted as sequences of stars pulsating in different modes. This is not observed in Fornax or in Carina since both galaxies present a more 

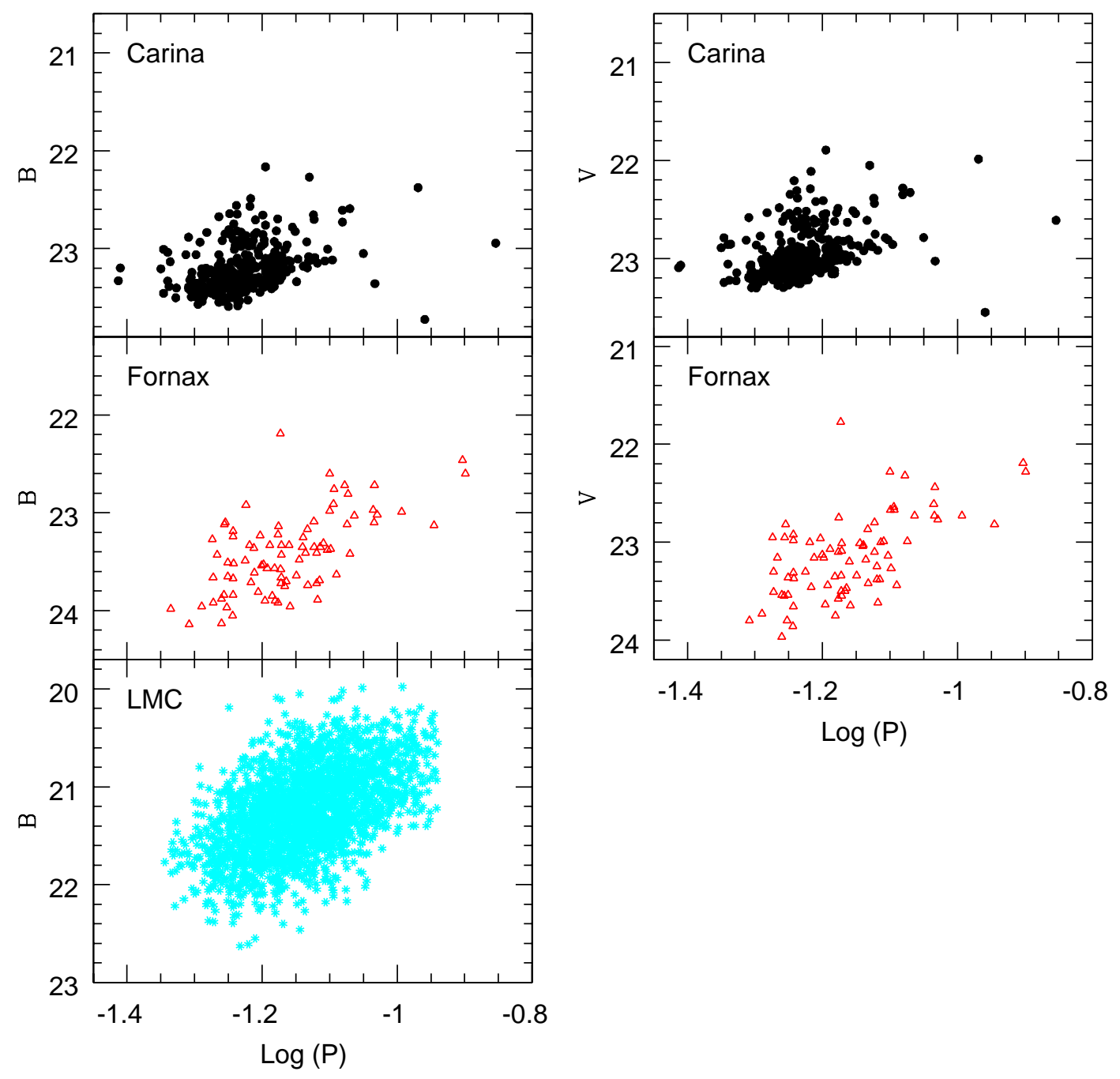

Fig. 18.- Observed period-luminosity diagrams in the $\mathrm{B}$ and $\mathrm{V}$ bands for Carina (top panels), Fornax (middle panels) and the LMC (lower panel). Data for Fornax and the LMC are from Poretti et al. (2008) and Garg et al. (2010), respectively. For a better comparison, the vertical axis scale in all panels is the same. 
flattened distribution of projected distances on the P-L relationship. No clear peaks are present in the histograms for these galaxies.

A spread in metallicity and/or a significant depth along the line of sight may be possible explanations for these differences. The contribution of depth along the line of sight, however, should have only a small contribution in the observed spread in brightness, at least in the case of Fornax. A spread of $5 \mathrm{kpc}$ along the line of sight in Fornax (which is about its angular size) should produce a spread in magnitude of only 0.02 mags at the distance of this galaxy $(120 \mathrm{kpc})$. This is too small to be responsible of filling up the gap between the two sequences of pulsators. A spread in metallicity could have a larger effect. From equations 6 and 7, it is inferred that a spread of $1.0 \mathrm{dex}$ in $[\mathrm{Fe} / \mathrm{H}]$ would produce variations in absolute magnitude of the order of $0.2-0.3$ mags, which are large enough to account for the spread observed in Fornax and the LMC. Spectroscopy of Fornax shows that the galaxy has a significant metallicity gradient (Pont et al. 2004), which is probably not the case for Carina. Bono et al. (2010) argued that the intermediate age population of Carina has only a modest metallicity spread of $\sim 0.5$ dex at most.

A third difference among the DC population in these three galaxies is the slope of the PL relationship. The vertical axis in Figure 18 has the same scale for the three galaxies. It is clear that the PL relationship in both Fornax and the LMC have a steeper slope than Carina. Indeed, Poretti et al. (2008) fits the F pulsators in Fornax with a slope of -3.3 (in the V band), while Garg et al. (2010) found a slope of -3.43 in the LMC (in the broadband VR filter). The slope we find in Carina $(-1.68$ in V) is significantly shallower. Part of the difference may be due to the fact that the narrow range of periods found among the Carina DC stars may not provide a strong constraint on the slope of the PL relationship. But the effect of age and metallicity spreads within each galaxy on the P-L relationship slope remains to be studied.

From these comparisons it is clear that the DC population in these three systems show differences that may be important to understand the evolution and star formation in these galaxies. All three are known to have a complex mix of stellar populations, but their star formation histories are likely quite distinct. Carina has produced a large number of DC stars, but they have narrower properties that may indicate that they have a common origin. Presumably, most of them are main sequence stars from the populous intermediate-age population known to exist in this galaxy (Monelli et al. 2003). On the other hand, Fornax, which also has a prominent intermediate-age population, has DC stars with broader properties (both in period and luminosity). A significant spread of metallicities in the intermediate age population of Fornax may contribute to their different mean properties. Another possibility, which was suggested by Poretti et al. (2008) is that the DC sample in Fornax contains 
a mixture of stars arising from different evolutionary paths. Blue stragglers from the old population in that galaxy may also populate the instability strip in this galaxy. However, Carina also has an old population (it has numerous RR Lyrae stars, as shown above), and thus it may also contain, in principle, a combination of main sequence and blue stragglers among its DC stars. If so, the properties of both type of stars have very similar properties in Carina, which is not the case for Fornax. Alternatively, the larger spread observed among the Fornax and LMC DC stars may be a reflection of the contribution of old stars to the total population in these galaxies. CMD studies show that in Carina the old population may be as little as 10\% (Hurley-Keller et al. 1998), while it is significantly higher in Fornax and the LMC (Mateo 1998; Grebel 1999; Coleman \& de Jong 2008). Detailed comparisons with stellar evolutionary models may help to understand the origin and properties of DC stars under different conditions.

\section{The Extended Spatial Distribution of the Variable Stars in Carina}

Figure 10 shows that there are RR Lyrae stars, anomalous Cepheids and DCs well outside the King (1962) tidal radius of Carina (Irwin \& Hatzidimitriou 1995). The existence of these stars is additional proof of the tidal disruption of Carina. We detected DC stars up to a distance of $55^{\prime}$ of the center of Carina. Similarly, RR Lyrae stars and anomalous Cepheids were detected up to $65^{\prime}$ and $75^{\prime}$ respectively. The presence of these stars at such large distances is consistent with the results described in Battaglia et al. (2012) in which both the old and intermediate age population of Carina extends to distances of $\sim 60^{\prime}$. It would be very unlikely that these stars were actually field stars in the Milky Way since very few halo stars are expected at such large distances from the galactic center.

Since both DC and RR Lyrae stars are standard candles and they both extend to large distances from the center of Carina, it is natural to ask if there is a gradient in distance from one side of the galaxy to the other. Indeed, the best P-L relationship fitted to our data (Fig 15) shows some dispersion which could be due, at least in part, to differences in depth along the line of sight of the DC stars. In order to investigate this issue, we calculated $\Delta V=V_{0}-V_{F}$ for all the DC F-pulsators, where $V_{F}$ is the magnitude predicted by the P-L relationship fitted to the data (eq. 5). In the top panel of figure 19, we show $\Delta V$ as a function of $r^{\prime}$, the projected distance of each star on the semi-major axis of the galaxy (assuming a position angle of $65^{\circ}$, Irwin \& Hatzidimitriou 1995). In this plot, negative values of $r^{\prime}$ refer to the N-W side of Carina. It is clear that there is no trend of distance with position in the galaxy. A similar conclusion is reached by looking at the variation of the magnitude of the RR Lyrae stars as a function of $r^{\prime}$ (bottom panel in Figure 19). No gradient is evident in 


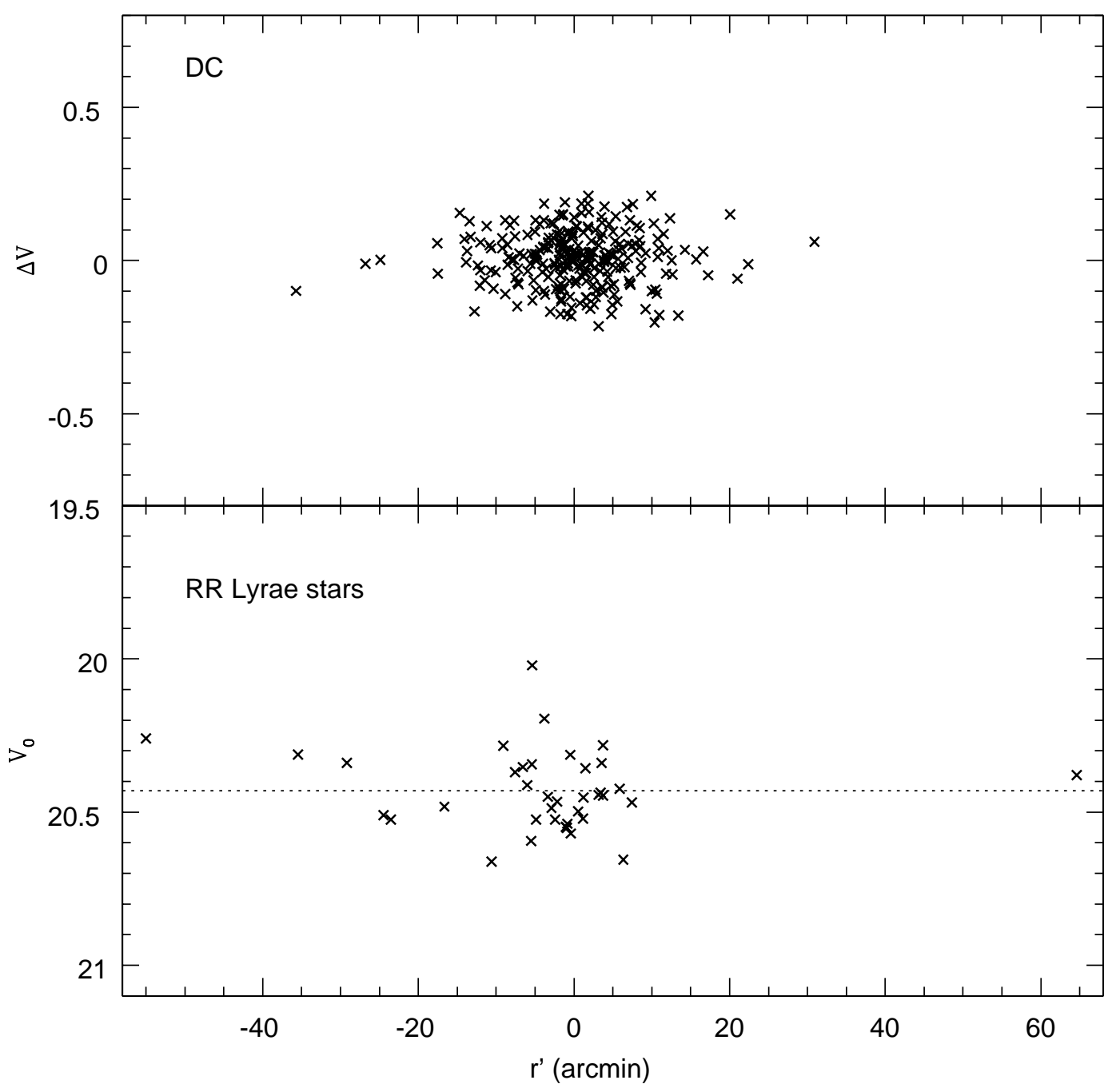

Fig. 19.- (Top): Variation of $\Delta V=V_{0}-V_{F}$ along $r^{\prime}$, the projected distance on the semimajor axis, for all DC stars identified as F pulsators. (Bottom): Variation of $V_{0}$ along $r^{\prime}$ for all RR Lyrae stars. The dotted line indicates the value of the mean reddening-corrected $V$ magnitude of all the RR Lyrae. 
the extinction corrected $\mathrm{V}$ magnitude of the RR Lyraes stars along their projected distance on the semi-major axis of the galaxy.

The dispersion of the P-L relationship of the DC in the $\mathrm{V}$ band (eq 5) is 0.09 mags. This implies a fractional error in distance $\left(\sigma_{d} / d\right)$ of only $4 \%$. However, at the distance of Carina, a fractional error of $4 \%$ implies $\sigma_{d} \sim 4 \mathrm{kpc}$. The projected size of Carina is only $1.3 \mathrm{kpc}$ and thus it would have been impossible to measure any internal sub-structure in the galaxy using these stars unless Carina were very much more extended along the light of sight than in the transverse direction. The dispersion observed in the P-L relationship of the DC puts an upper limit in the size of a gradient between the more distant DC stars detected on both sides of the galaxy, $\Delta d / \Delta r^{\prime}$. If such gradient exists it should not be larger than 4 $\mathrm{kpc} / 67^{\prime}=0.06 \mathrm{kpc} /{ }^{\prime}$. A clear limitation of this conclusion is of course the small number of stars at large $\left|r^{\prime}\right|$.

\section{The frequency of the Dwarf Cepheids}

In order to estimate the frequency of DC stars in Carina, we selected the central region of the galaxy in which we have the overlap region of Fields 3 and $4\left(100.25<\alpha\left(^{\circ}\right)<100.53\right.$, $-51.17<\delta\left(^{\circ}\right)<-50.77$ ). This region has also a large number of epochs (see Figure 1) and hence our completeness is likely to be very high in this region. We selected stars in the upper part of the main sequence, namely stars within the following boundaries: $22.5<V<23.3$ and $0.15<(B-V)<0.35$. There are 2,531 stars in this part of the CMD and 197, or $8 \%$ of them, are DC stars. This figure is higher than the one provided by Mateo et al. (1998), 2\%. However, as in Mateo et al. (1998), our figure of $8 \%$ should also be considered a lower limit in the number of DC stars produced in Carina since most likely we are missing small amplitude variables. With a much lower detection threshold for the amplitude of the variables Balona \& Dziembowski (2011) argue that no more than 50\% of the main sequence stars in the Kepler field are pulsating. In contrast, the frequency of pulsation at the RR Lyrae luminosities is nearly $100 \%$, and appears to be similarly high for anomalous Cepheids and classical Cepheids as well. The reason why not all the stars within the instability strip are DC stars (or at least DC stars with amplitudes greater than 0.2-0.4 mags) remains to be explained. Something about main sequence stars is able to suppress pulsation (surface convection, magnetic fields, rotation, higher surface gravity?) which is less effective or absent in more luminous pulsators.

Results from the Kepler mission suggest that DC of high amplitude are extremely rare among field stars (Balona \& Nemec 2012). Indeed, only one out of more than 1600 DC stars identified by Kepler varies with an amplitude $>0.3$ mags. This finding suggests two possible 
scenarios: either the high amplitude DC found in the extragalactic systems are just the tip of the iceberg of a much larger population which is below detection with current observations, or the properties of DCs in the galactic field are substantially different from the ones in extragalactic systems. The number of high amplitude DC stars in Carina suggests that the later is a more feasible option. If the frequency observed in the field were to hold in Carina, we would expect that for the 197 high amplitude DC in the central part of Carina, there should be $\sim 300,000$ low amplitude pulsators, which is $\sim 10^{2}$ times the total number of stars detected in that part of the CMD. Thus, the frequency of high amplitude DC stars in Carina is at least $100 \times$ higher than in the field.

\subsection{Specific frequency of DC in Local group galaxies and Galactic globular clusters}

We calculated the specific frequency of DC stars, $S_{\mathrm{DC}}$, by adapting the definition used by Mateo et al. (1995) for the specific frequency of anomalous Cepheids, that is, $S_{\mathrm{DC}}=N_{\mathrm{DC}} / L_{V}$, with the luminosity in units of $10^{5} L_{\odot}$. In the case of Carina we corrected the number of DC stars to include only stars inside the tidal radius (as given by Walcher et al. 2003). In the case of Fornax, we corrected the number of DC for the fact that the field observed by Poretti et al. (2008) did not cover the whole galaxy. We calculated the observed fraction of the luminosity of Fornax by integrating the King (1962) profile (see Mateo et al. 1995; Vivas \& Zinn 2006) with the parameters $r_{c}=13$ '.7 and $r_{t}=71$ '.1 (Irwin \& Hatzidimitriou 1995). The ellipticity and position angle of the galaxy were taken into account to determined the observed fraction of the galaxy, which turned out to be 0.88 . Thus, the estimated number of DC in Fornax is $85 / 0.88=97$. We did not attempt to correct the number of DC in the LMC given the complexity of the superMACHO dataset (Rest et al. 2005). However, that survey covers the entire central part of the LMC (hence, most of the light), and the specific frequency we calculated is likely close to the real value. Still, it should be considered just as an approximate value.

DC stars have been detected as well in several Galactic globular clusters. We selected the clusters with $\geq 5$ DC stars from the Catalog of Variable Stars in Globular Clusters by Clement et al. (2001). For the globular clusters M55 and NGC5466, we took the updated (June 2013) value of $N_{\mathrm{DC}}$ in Clement et al's catalog ${ }^{2}$, which includes more recent observations of those clusters by Pych et al. (2001) and Jeon et al. (2004).

The specific frequencies of DC for all these stellar systems are reported in Table 7.

\footnotetext{
${ }^{2}$ http://www.astro.utoronto.ca/ cclement/read.html
} 
We did not make any consideration for missing variables in any of these stellar systems. If incompleteness were an important issue, the specific frequencies would be higher than the ones we calculated here. Carina is the stellar system with the highest specific frequency of DC stars known to date. It has a significant higher specific frequency than the Fornax dSph galaxy and the LMC. It also have more DC stars (per luminosity) than all the globular clusters than have been searched for this type of variables.

\section{CONCLUSIONS}

We have searched the Carina dSph galaxy for DC stars, extending significantly the area first explored by Mateo et al. (1998). With 340 DC stars, we find that Carina is very rich in this type of stars which is likely linked to the fact that this galaxy has a significant intermediate age population and hence, a well populated main sequence in the region of overlap with the instability strip. It has the highest specific frequency of DC among the Local Group galaxies and galactic globular clusters that have been searched for this type of stars.

The DC stars in Carina spread from 1.2 to almost 3 magnitudes below the horizontal branch. Most of the stars (80\%), however, are faint and clump around $V \sim 23.1$ (or $\sim 2.5$ mags below the horizontal branch). These stars are identified with ones pulsating in the fundamental mode, which follow a tight P-L relationship. Using several calibrations of the P-L relationship for DC stars, we derived a distance modulus of $\mu_{0}=20.17 \pm 0.10$ which agrees very well with the distance derived by D03 using RR Lyrae stars.

The survey covers the whole galaxy and extends an angular distance of $\sim 100^{\prime}$ from the center of Carina along the semi-major axis, in both directions. Although our completeness for DC stars is low and not uniform in the most external parts of the survey, we discovered several DC stars located up to $\sim 1^{\circ}$ from the center which is equivalent to $\sim 2-3$ times the tidal radius of Carina (depending on the assumed tidal radius which vary from $22^{\prime} 5$ (Walcher et al. 2003) to $28^{\prime}$ (Irwin \& Hatzidimitriou 1995)). The existence of these extra-tidal stars in the Carina field is reinforced as well by the discovery of RR Lyrae stars and anomalous Cepheids at large distances from the center, and confirms previous findings that this galaxy is under tidal disruption. In particular, the fact that all three types of variables are found far away from the center confirms that both the old and intermediate age population in Carina extends beyond its tidal radius (see Battaglia et al. 2012).

The properties of DC stars in dSph galaxies reflect the different star formation histories of these galaxies and possibly their mean metallicities. Carina's DC stars differ significantly 
in several aspects with respect to the ones in Fornax and the LMC. The period distribution in Carina is narrower than in those other two galaxies. Also, the mean period in Carina is smaller. Only in Carina the sequence of fundamental pulsators is well separated from the first overtone one. We speculate that most of the DC stars in Carina come from a stellar population which has only a small spread in metallicity. In Fornax a large range of metallicity for the intermediate age population may be the reason for the larger spread observed in the diagram of brightness versus period. This possibility has grounds in the study of the populations of those galaxies with other methods. A detailed analysis of the CMD of Carina (Bono et al. 2010) and spectroscopy in Fornax (Pont et al. 2004) indicate that the spread in metallicity in Carina is probably very small, while that is not the case for Fornax. In the LMC, depth along the line of sight may also play a role in the observed properties of the DC stars. As in Carina, Fornax is also too far away for depth effects to likely have a measurable effect in the PL diagram.

Our findings in Carina suggest that DC stars are a good tool to find tidal tails of disrupting systems. They should be useful as well to find streams in the Milky Way's halo if they have an intermediate-age population associated with them. In Carina, DCs are $\sim 3-4$ times more numerous than RR Lyrae stars, although that ratio may be different in other systems depending on their particular combination of stellar populations.

Further observations of DC stars in other extragalactic systems and comparison with existing and updated theoretical models of stellar evolution and stellar pulsation will help to shed more light on their origin and their production under different conditions of age and chemical composition.

Based on observations obtained at the Cerro Tololo Inter-American Observatory, National Optical Astronomical Observatories, operated by the Association of Universities for Research in Astronomy (AURA) under cooperative agreement with the National Science Foundation. AKV thanks the hospitality of the Department of Astronomy at University of Michigan during her sabbatical leave in which most of this work was made.

Facility: Blanco

\section{A. Lightcurves}

The full set of light curves are presented as online-only material in Figures 20.1-20.4 (RR Lyrae stars), 21.1-21.29 (DC stars), 22.1 (anomalous Cepheids), and 23.1 (Miscellaneous periodic variable stars). 
Fig. Set 20. RR Lyrae Stars

Fig. Set 21. Dwarf Cepheid Stars

Fig. Set 22. Anomalous Cepheid Stars

Fig. Set 23. Miscellaneous Stars

\section{REFERENCES}

Balona, L. A., \& Dziembowski, W. A. 2011, MNRAS, 417, 591

Balona, L. A., \& Nemec, J. M. 2012, MNRAS, 426, 2413

Battaglia, G., Irwin, M., Tolstoy, E., de Boer, T., \& Mateo, M. 2012, ApJ, 761, L31

Binney, J., \& Merrifield, M. 1998, Galactic Astronomy

Bono, G., Caputo, F., Santolamazza, P., Cassisi, S., \& Piersimoni, A. 1997, AJ, 113, 2209

Bono, G. et al. 2010, PASP, 122, 651

Breger, M. 2000, in Astronomical Society of the Pacific Conference Series, Vol. 210, Delta Scuti and Related Stars, ed. M. Breger \& M. Montgomery, 3

Clement, C. M. et al. 2001, AJ, 122, 2587

Cohen, R. E., \& Sarajedini, A. 2012, MNRAS, 419, 342

Coleman, M. G., \& de Jong, J. T. A. 2008, ApJ, 685, 933

Dall'Ora, M. et al. 2003, AJ, 126, 197

Fiorentino, G., Stetson, P. B., Monelli, M., Bono, G., Bernard, E. J., \& Pietrinferni, A. 2012, ApJ, 759, L12

Garg, A. et al. 2010, AJ, 140, 328

Grebel, E. K. 1999, in IAU Symposium, Vol. 192, The Stellar Content of Local Group Galaxies, ed. P. Whitelock \& R. Cannon, 17

Grebel, E. K. 2011, in EAS Publications Series, Vol. 48, EAS Publications Series, ed. M. Koleva, P. Prugniel, \& I. Vauglin, 315-327

Harris, W. E. 1996, AJ, 112, 1487 
Helmi, A. et al. 2006, ApJ, 651, L121

Hurley-Keller, D., Mateo, M., \& Nemec, J. 1998, AJ, 115, 1840

Irwin, M., \& Hatzidimitriou, D. 1995, MNRAS, 277, 1354

Jeon, Y.-B., Lee, M. G., Kim, S.-L., \& Lee, H. 2004, AJ, 128, 287

Kinemuchi, K., Harris, H. C., Smith, H. A., Silbermann, N. A., Snyder, L. A., La Cluyzé, A. P., \& Clark, C. L. 2008, AJ, 136, 1921

King, I. 1962, AJ, 67, 471

Koch, A., Grebel, E. K., Wyse, R. F. G., Kleyna, J. T., Wilkinson, M. I., Harbeck, D. R., Gilmore, G. F., \& Evans, N. W. 2006, AJ, 131, 895

Kuehn, C. et al. 2008, ApJ, 674, L81

Lafler, J., \& Kinman, T. D. 1965, ApJS, 11, 216

Majewski, S. R., Ostheimer, J. C., Patterson, R. J., Kunkel, W. E., Johnston, K. V., \& Geisler, D. 2000, AJ, 119, 760

Mateo, M. 1993, in Astronomical Society of the Pacific Conference Series, Vol. 53, Blue Stragglers, ed. R. A. Saffer, 74

Mateo, M., Fischer, P., \& Krzeminski, W. 1995, AJ, 110, 2166

Mateo, M., Hurley-Keller, D., \& Nemec, J. 1998, AJ, 115, 1856

Mateo, M. L. 1998, ARA\&A, 36, 435

Mateu, C., Vivas, A. K., Downes, J. J., Briceño, C., Zinn, R., \& Cruz-Diaz, G. 2012, MNRAS, 427, 3374

McNamara, D. H. 2011, AJ, 142, 110

Monelli, M. et al. 2003, AJ, 126, 218

Muñoz, R. R. et al. 2006, ApJ, 649, 201

Nemec, J. M., Nemec, A. F. L., \& Lutz, T. E. 1994, AJ, 108, 222

Pont, F., Zinn, R., Gallart, C., Hardy, E., \& Winnick, R. 2004, AJ, 127, 840

Poretti, E. et al. 2008, ApJ, 685, 947 
Pych, W., Kaluzny, J., Krzeminski, W., Schwarzenberg-Czerny, A., \& Thompson, I. B. 2001, A\&A, 367, 148

Rest, A. et al. 2005, ApJ, 634, 1103

Rizzi, L., Held, E. V., Bertelli, G., \& Saviane, I. 2003, ApJ, 589, L85

Rizzi, L., Held, E. V., Saviane, I., Tully, R. B., \& Gullieuszik, M. 2007, MNRAS, 380, 1255

Saha, A., Monet, D. G., \& Seitzer, P. 1986, AJ, 92, 302

Schechter, P. L., Mateo, M., \& Saha, A. 1993, PASP, 105, 1342

Schlegel, D. J., Finkbeiner, D. P., \& Davis, M. 1998, ApJ, 500, 525

Smecker-Hane, T. A., Stetson, P. B., Hesser, J. E., \& Vandenberg, D. A. 1996, in Astronomical Society of the Pacific Conference Series, Vol. 98, From Stars to Galaxies: the Impact of Stellar Physics on Galaxy Evolution, ed. C. Leitherer, U. Fritze-vonAlvensleben, \& J. Huchra, 328

Smith, H. A. 1995, RR Lyrae stars (Cambridge Astrophysics Series, Cambridge, New York: Cambridge University Press, —c1995)

Sollima, A., Cacciari, C., Bellazzini, M., \& Colucci, S. 2010, MNRAS, 406, 329

Stetson, P. B. 1996, PASP, 108, 851

Vivas, A. K., \& Zinn, R. 2006, AJ, 132, 714

Vivas, A. K. et al. 2004, AJ, 127, 1158

Walcher, C. J., Fried, J. W., Burkert, A., \& Klessen, R. S. 2003, A\&A, 406, 847

Watkins, L. L. et al. 2009, MNRAS, 398, 1757

Zinn, R., \& Searle, L. 1976, ApJ, 209, 734 
Table 1. Coordinates and Number of Repeated Observations in the Carina Fields

\begin{tabular}{cccrrr}
\hline \hline Field & $\alpha(2000.0)$ & $\delta(2000.0)$ & $N_{V}$ & $N_{B}$ & $N_{\text {nights }}$ \\
\hline 1 & $06: 34: 24.2$ & $-51: 29: 15$ & 13 & 9 & 2 \\
2 & $06: 37: 15.3$ & $-51: 16: 35$ & 12 & 10 & 3 \\
3 & $06: 40: 12.7$ & $-51: 04: 39$ & 14 & 9 & 3 \\
4 & $06: 42: 58.8$ & $-50: 52: 24$ & 12 & 9 & 3 \\
5 & $06: 45: 51.4$ & $-50: 38: 28$ & 12 & 9 & 2 \\
6 & $06: 48: 46.7$ & $-50: 26: 37$ & 13 & 9 & 3 \\
7 & $06: 43: 00.4$ & $-51: 04: 33$ & 2 & 2 & 1 \\
8 & $06: 40: 14.9$ & $-50: 52: 21$ & 2 & 2 & 1 \\
\hline
\end{tabular}


Table 2. Properties of the RR Lyrae Stars

\begin{tabular}{|c|c|c|c|c|c|c|c|c|c|c|c|c|c|c|}
\hline ID & $\begin{array}{l}\mathrm{RA}(2000.0) \\
\quad(\mathrm{deg})\end{array}$ & $\begin{array}{c}\operatorname{DEC}(2000.0) \\
\quad(\mathrm{deg})\end{array}$ & $\langle B\rangle$ & $\langle V\rangle$ & Amp B & Amp V & $N_{B}$ & $N_{V}$ & $\begin{array}{l}\text { Period } \\
\text { (d) }\end{array}$ & $\mathrm{E}(\mathrm{B}-\mathrm{V})$ & Type & ID $(\mathrm{D} 03)^{\mathrm{a}}$ & Type $(\mathrm{D} 03)^{\mathrm{a}}$ & $\begin{array}{c}\operatorname{Per}(\mathrm{D} 03)^{\mathrm{a}} \\
(\mathrm{d})\end{array}$ \\
\hline RRL-1 & 99.035133 & -51.293030 & 20.94 & 20.55 & 0.55 & 0.45 & 16 & 20 & 0.629 & 0.088 & $\mathrm{ab}$ & $\ldots$ & $\ldots$ & $\ldots$ \\
\hline RRL-2 & 99.577423 & -51.253571 & 21.04 & 20.60 & 0.83 & 0.65 & 19 & 26 & 0.523 & 0.089 & $a b$ & $\ldots$ & $\ldots$ & $\ldots$ \\
\hline RRL-3 & 99.709419 & -51.181721 & 21.06 & 20.62 & 0.56 & 0.46 & 19 & 24 & 0.544 & 0.088 & $a b$ & $\ldots$ & $\ldots$ & $\ldots$ \\
\hline RRL-4 & 99.731697 & -51.024300 & 21.32 & 20.85 & 0.17 & 0.24 & 17 & 24 & 0.204 & 0.105 & $\mathrm{c}$ & $\ldots$ & $\cdots$ & $\cdots$ \\
\hline RRL-5 & 99.855621 & -51.156158 & 21.27 & 20.82 & 0.14 & 0.17 & 11 & 15 & 0.107 & 0.090 & c & $\ldots$ & $\ldots$ & $\ldots$ \\
\hline RRL-6 & 100.041039 & -51.134750 & 21.21 & 20.78 & 0.35 & 0.29 & 11 & 16 & 0.250 & 0.092 & $\mathrm{c}$ & V189 & $a b$ & 0.700 \\
\hline RRL-7 & 100.122673 & -51.004780 & 21.30 & 20.95 & 0.36 & 0.41 & 11 & 15 & 0.146 & 0.089 & $\mathrm{c}$ & V151 & $\mathrm{c}$ & 0.343 \\
\hline RRL-8 & 100.132042 & -50.811119 & 20.49 & 20.35 & 0.72 & 0.56 & 11 & 16 & 0.245 & 0.101 & $\mathrm{c}$ & V148 & c & 0.324 \\
\hline RRL-9 & 100.154999 & -50.990261 & 20.82 & 20.57 & 0.52 & 0.35 & 11 & 16 & 0.246 & 0.088 & $\mathrm{c}$ & V144 & $\mathrm{c}$ & 0.391 \\
\hline RRL-10 & 100.222366 & -50.981449 & 20.84 & 20.63 & 0.55 & 0.47 & 11 & 16 & 0.303 & 0.087 & $\mathrm{c}$ & V125 & $\mathrm{ab}$ & 0.597 \\
\hline RRL-11 & 100.273499 & -51.007790 & 21.32 & 20.86 & 0.56 & 0.57 & 21 & 27 & 0.679 & 0.083 & $a b$ & V116 & $\mathrm{ab}$ & 0.685 \\
\hline RRL-12 & 100.293961 & -51.056358 & 20.92 & 20.67 & 0.68 & 0.51 & 21 & 28 & 0.281 & 0.080 & $\mathrm{c}$ & V198 & $\mathrm{d}$ & 0.402 \\
\hline RRL-13 & 100.297867 & -51.037788 & 21.04 & 20.61 & 0.62 & 0.55 & 19 & 27 & 0.663 & 0.081 & $\mathrm{ab}$ & V196 & $a b$ & 0.670 \\
\hline RRL-14 & 100.318878 & -51.150841 & 20.94 & 20.62 & 0.84 & 0.65 & 21 & 28 & 0.630 & 0.076 & $\mathrm{ab}$ & V105 & $\mathrm{ab}$ & 0.630 \\
\hline RRL-15 & 100.336128 & -51.008739 & 21.05 & 20.71 & 0.80 & 0.61 & 21 & 29 & 0.719 & 0.081 & $\mathrm{ab}$ & V192 & $\mathrm{d}$ & 0.390 \\
\hline RRL-16 & 100.359253 & -50.992741 & 21.14 & 20.73 & 0.47 & 0.42 & 20 & 26 & 0.651 & 0.082 & $a b$ & V191 & $a b$ & 0.667 \\
\hline RRL-17 & 100.364998 & -50.933701 & 20.86 & 20.59 & 0.83 & 0.66 & 22 & 29 & 0.617 & 0.087 & $\mathrm{ab}$ & V183 & $a b$ & 0.611 \\
\hline RRL-18 & 100.369789 & -51.114010 & 21.12 & 20.76 & 0.61 & 0.47 & 22 & 29 & 0.628 & 0.073 & $\mathrm{ab}$ & V92 & $\mathrm{ab}$ & 0.620 \\
\hline RRL-19 & 100.372803 & -51.074558 & 20.76 & 20.44 & 0.43 & 0.32 & 14 & 17 & 0.715 & 0.075 & $\mathrm{ab}$ & V91 & $\mathrm{ab}$ & 0.720 \\
\hline RRL-20 & 100.374657 & -50.869629 & 21.02 & 20.67 & 0.77 & 0.66 & 20 & 27 & 0.643 & 0.096 & $\mathrm{ab}$ & V90 & $\mathrm{ab}$ & 0.618 \\
\hline RRL-21 & 100.374786 & -50.787121 & 21.00 & 20.68 & 0.80 & 0.61 & 22 & 29 & 0.383 & 0.104 & $\mathrm{ab}$ & V89 & $\mathrm{d}$ & 0.385 \\
\hline RRL-22 & 100.398537 & -50.835381 & 21.23 & 20.77 & 0.34 & 0.34 & 22 & 28 & 0.638 & 0.101 & $\mathrm{ab}$ & V85 & $\mathrm{ab}$ & 0.644 \\
\hline RRL-23 & 100.413544 & -51.094349 & 20.93 & 20.72 & 1.32 & 1.05 & 21 & 30 & 0.602 & 0.073 & $a b$ & V77 & $a b$ & 0.605 \\
\hline RRL-24 & 100.420128 & -51.026020 & 21.23 & 20.80 & 0.66 & 0.56 & 20 & 27 & 0.620 & 0.077 & $a b$ & V195 & $a b$ & 0.628 \\
\hline RRL-25 & 100.428787 & -50.980412 & 20.96 & 20.76 & 1.42 & 1.17 & 22 & 30 & 0.595 & 0.081 & $a b$ & $\ldots$ & $\cdots$ & $\ldots$ \\
\hline RRL-26 & 100.444794 & -50.977589 & 21.19 & 20.78 & 0.52 & 0.52 & 22 & 30 & 0.669 & 0.081 & $\mathrm{ab}$ & V200 & $a b$ & 0.624 \\
\hline RRL-27 & 100.445541 & -51.118710 & 21.20 & 20.76 & 0.94 & 0.87 & 22 & 30 & 0.566 & 0.072 & $\mathrm{ab}$ & V73 & $\mathrm{ab}$ & 0.571 \\
\hline RRL-28 & 100.454826 & -50.988541 & 21.12 & 20.71 & 0.57 & 0.48 & 22 & 30 & 0.664 & 0.080 & $\mathrm{ab}$ & V68 & $\mathrm{ab}$ & 0.675 \\
\hline RRL-29 & 100.455872 & -50.903111 & 21.14 & 20.72 & 0.29 & 0.21 & 22 & 29 & 0.394 & 0.088 & $\mathrm{c}$ & V179 & $\mathrm{ab}$ & 0.665 \\
\hline RRL-30 & 100.469383 & -51.088799 & 21.06 & 20.77 & 0.78 & 0.62 & 22 & 30 & 0.601 & 0.073 & $a b$ & V67 & $a b$ & 0.613 \\
\hline RRL-31 & 100.482826 & -50.926441 & 20.94 & 20.56 & 0.99 & 0.80 & 19 & 25 & 0.650 & 0.085 & $\mathrm{ab}$ & V65 & $a b$ & 0.642 \\
\hline RRL-32 & 100.492867 & -50.793819 & 21.12 & 20.80 & 0.59 & 0.46 & 20 & 21 & 0.625 & 0.102 & $\mathrm{ab}$ & V61 & $\mathrm{ab}$ & 0.624 \\
\hline RRL-33 & 100.499001 & -51.110691 & 21.30 & 20.80 & 0.72 & 0.76 & 22 & 30 & 0.603 & 0.072 & $\mathrm{ab}$ & V60 & $\mathrm{ab}$ & 0.615 \\
\hline
\end{tabular}


Table 2-Continued

\begin{tabular}{|c|c|c|c|c|c|c|c|c|c|c|c|c|c|c|}
\hline ID & $\begin{array}{l}\mathrm{RA}(2000.0) \\
\quad(\operatorname{deg})\end{array}$ & $\begin{array}{c}\mathrm{DEC}(2000.0) \\
(\mathrm{deg})\end{array}$ & $\langle B\rangle$ & $\langle V\rangle$ & Amp B & Amp V & $N_{B}$ & $N_{V}$ & $\begin{array}{l}\text { Period } \\
\quad(d)\end{array}$ & $\mathrm{E}(\mathrm{B}-\mathrm{V})$ & Type & ID $(\mathrm{D} 03)^{\mathrm{a}}$ & Type $(\mathrm{D} 03)^{\mathrm{a}}$ & $\begin{array}{l}\text { Per }(\text { D03) } \\
\quad(d)\end{array}$ \\
\hline RRL-34 & 100.504372 & -50.955441 & 21.01 & 20.71 & 0.92 & 0.81 & 22 & 30 & 0.617 & 0.081 & $\mathrm{ab}$ & $\cdots$ & $\cdots$ & $\ldots$ \\
\hline RRL-35 & 100.511917 & -50.882431 & 21.00 & 20.71 & 0.93 & 0.77 & 22 & 30 & 0.610 & 0.087 & $\mathrm{ab}$ & V57 & $\mathrm{ab}$ & 0.612 \\
\hline RRL-36 & 100.517754 & -51.161480 & 21.04 & 20.78 & 1.24 & 1.06 & 21 & 30 & 0.586 & 0.070 & $\mathrm{ab}$ & V206 & $\mathrm{ab}$ & 0.585 \\
\hline RRL-37 & 100.538002 & -50.898159 & 21.22 & 20.93 & 0.34 & 0.27 & 12 & 14 & 0.246 & 0.085 & $\mathrm{c}$ & V47 & $\mathrm{c}$ & 0.324 \\
\hline RRL-38 & 101.874420 & -50.419701 & 21.18 & 20.77 & 0.41 & 0.31 & 16 & 23 & 0.189 & 0.122 & c & $\cdots$ & $\cdots$ & $\ldots$ \\
\hline
\end{tabular}

a Data from Dall'Ora et al. (2003). 
Table 3. Properties of the anomalous Cepheid Stars

\begin{tabular}{|c|c|c|c|c|c|c|c|c|c|c|c|c|}
\hline ID & $\begin{array}{l}\mathrm{RA}(2000.0) \\
\quad(\operatorname{deg})\end{array}$ & $\begin{array}{l}\operatorname{DEC}(2000.0) \\
\quad(\operatorname{deg})\end{array}$ & $\langle B\rangle$ & $\langle V\rangle$ & Amp B & Amp V & $N_{B}$ & $N_{V}$ & $\begin{array}{l}\text { Period } \\
\text { (d) }\end{array}$ & $\mathrm{E}(\mathrm{B}-\mathrm{V})$ & ID $(\mathrm{D} 03)^{\mathrm{a}}$ & $\begin{array}{c}\text { Per }(\text { D03) } \\
\text { (d) }\end{array}$ \\
\hline AC-1 & 99.185699 & -51.036240 & 20.44 & 19.95 & 0.21 & 0.20 & 10 & 12 & 0.186 & 0.045 & $\ldots$ & $\ldots$ \\
\hline $\mathrm{AC}-2$ & 100.367668 & -51.012611 & 19.52 & 19.28 & 0.25 & 0.24 & 22 & 28 & 0.444 & 0.061 & V193 & 0.424 \\
\hline $\mathrm{AC}-3$ & 100.386169 & -50.950211 & 19.00 & 18.73 & 0.43 & 0.40 & 19 & 24 & 0.859 & 0.060 & V87 & 0.880 \\
\hline $\mathrm{AC}-4$ & 100.415833 & -50.983921 & 19.53 & 19.20 & 1.38 & 1.19 & 22 & 30 & 0.545 & 0.063 & V190 & 1.160 \\
\hline $\mathrm{AC}-5$ & 100.433540 & -50.838100 & 19.29 & 18.99 & 0.95 & 0.77 & 22 & 30 & 0.502 & 0.059 & V178 & 0.507 \\
\hline AC-6 & 100.483871 & -50.989330 & 19.25 & 19.09 & 0.51 & 0.72 & 20 & 29 & 0.312 & 0.066 & V203 & 0.467 \\
\hline $\mathrm{AC}-7$ & 100.602158 & -51.044540 & 19.89 & 19.68 & 0.19 & 0.16 & 11 & 14 & 0.197 & 0.070 & V205 & 0.383 \\
\hline AC- 8 & 100.642174 & -50.972210 & 19.04 & 18.78 & 0.55 & 0.39 & 10 & 12 & 0.161 & 0.066 & $\ldots$ & $\ldots$ \\
\hline AC-9 & 101.818748 & -50.474560 & 19.21 & 19.13 & 0.36 & 0.55 & 8 & 23 & 0.476 & 0.091 & $\ldots$ & $\ldots$ \\
\hline AC-10 & 102.226418 & -50.509750 & 19.71 & 19.35 & 0.18 & 0.32 & 9 & 13 & 0.163 & 0.089 & $\ldots$ & $\ldots$ \\
\hline
\end{tabular}

àData from Dall'Ora et al. (2003). 
Table 4. Properties of the Dwarf Cepheid Stars

\begin{tabular}{|c|c|c|c|c|c|c|c|c|c|c|c|c|c|c|}
\hline ID & $\begin{array}{l}\mathrm{RA}(2000.0) \\
\quad(\mathrm{deg})\end{array}$ & $\begin{array}{c}\operatorname{DEC}(2000.0) \\
(\mathrm{deg})\end{array}$ & $\langle B\rangle$ & $\langle V\rangle$ & Amp B & Amp V & $N_{B}$ & $N_{V}$ & $\begin{array}{l}\text { Period } \\
\text { (d) }\end{array}$ & $\begin{array}{l}\text { Alt Period } \\
\text { (d) }\end{array}$ & $\mathrm{E}(\mathrm{B}-\mathrm{V})$ & Mode & ID $\left(\right.$ M98) ${ }^{\mathrm{a}}$ & $\begin{array}{c}\text { Per }(\text { M98 })^{\mathrm{a}} \\
(\mathrm{d})\end{array}$ \\
\hline DC-44 & 100.226624 & -51.024540 & 22.27 & 22.05 & 0.50 & 0.57 & 11 & 14 & 0.07410 & $\ldots$ & 0.054 & $\ldots$ & $\ldots$ & $\ldots$ \\
\hline DC-45 & 100.231827 & -51.011921 & 23.41 & 23.06 & 0.56 & 0.60 & 8 & 15 & 0.05779 & 0.05950 & 0.054 & $\mathrm{~F}$ & V4 & 0.05991 \\
\hline DC-46 & 100.240211 & -50.935970 & 23.02 & 22.76 & 0.42 & 0.38 & 9 & 16 & 0.06229 & $\ldots$ & 0.054 & $\ldots$ & $\ldots$ & $\ldots$ \\
\hline DC-47 & 100.241219 & -51.035511 & 23.23 & 22.91 & 0.64 & 0.68 & 9 & 15 & 0.05629 & $\ldots$ & 0.055 & $\mathrm{~F}$ & $\ldots$ & $\ldots$ \\
\hline DC-48 & 100.246582 & -50.828979 & 23.24 & 23.07 & 0.59 & 0.42 & 11 & 16 & 0.05489 & $\ldots$ & 0.053 & $\mathrm{~F}$ & $\ldots$ & $\ldots$ \\
\hline
\end{tabular}

${ }^{\text {a}}$ Data from Mateo et al. (1998).

Note. - Table 4 is published in its entirety in the electronic edition of the journal. A portion is shown here for guidance regarding its form and content. 
Table 5. Properties of the Miscellaneous Periodic Variable Stars

\begin{tabular}{lccccccccccc}
\hline \hline ID & $\begin{array}{c}\text { RA(2000.0) } \\
(\mathrm{deg})\end{array}$ & $\begin{array}{c}\mathrm{DEC}(2000.0) \\
(\mathrm{deg})\end{array}$ & $\langle B\rangle$ & $\langle V\rangle$ & Amp B & Amp V & $N_{B}$ & $N_{V}$ & $\begin{array}{c}\text { Period } \\
(\mathrm{d})\end{array}$ & $\mathrm{E}(\mathrm{B}-\mathrm{V})$ & Comment \\
& & & & & & & & & & & \\
\hline Mis-1 & 99.709000 & -51.342861 & 21.56 & 21.13 & 0.19 & 0.16 & 19 & 24 & 0.144 & 0.047 & \\
Mis-2 & 100.265793 & -50.914619 & 22.40 & 22.36 & 0.37 & 0.34 & 18 & 24 & 0.234 & 0.054 & \\
Mis-3 & 100.276123 & -50.924320 & 22.94 & 22.78 & 0.46 & 0.47 & 18 & 26 & 0.245 & 0.055 & \\
Mis-4 & 100.291924 & -50.987759 & 23.71 & 23.27 & 0.60 & 0.63 & 16 & 22 & 0.227 & 0.056 & Eclipsing Binary \\
Mis-5 & 100.322502 & -51.095890 & 22.01 & 21.82 & 0.38 & 0.35 & 21 & 29 & 0.204 & 0.061 & \\
Mis-6 & 100.327461 & -50.826900 & 23.71 & 23.49 & 0.57 & 0.49 & 20 & 24 & 0.216 & 0.054 & \\
Mis-7 & 100.421043 & -50.957130 & 23.25 & 23.16 & 0.41 & 0.40 & 17 & 25 & 0.173 & 0.062 & \\
Mis-8 & 101.777763 & -50.612900 & 23.63 & 23.49 & 0.88 & 0.87 & 12 & 18 & 0.238 & 0.086 & \\
Mis-9 & 101.782082 & -50.743198 & 23.94 & 23.36 & 0.72 & 0.54 & 7 & 17 & 0.193 & 0.098 \\
\hline
\end{tabular}


Table 6. Dereddened Distance Modulus for Carina using different P-L relationships for DC stars

\begin{tabular}{lccc}
\hline \hline \multicolumn{1}{c}{ P-L relationship } & {$[\mathrm{Fe} / \mathrm{H}]$} & $\mu_{0}$ & $\mathrm{rms}$ \\
\hline Nemec et al. (1994) & -2.0 & 19.99 & 0.10 \\
& -1.7 & 19.89 & 0.10 \\
Poretti et al. (2008) & & 20.20 & 0.13 \\
McNamara (2011) & -2.0 & 20.18 & 0.10 \\
& -1.7 & 20.24 & 0.10 \\
Cohen \& Sarajedini (2012) & & 20.33 & 0.12 \\
\hline Mean (for [Fe/H]=-1.7) & & 20.17 & 0.10 \\
\hline
\end{tabular}


Table 7. Specific Frequency of DC in Local Group objects and globular clusters

\begin{tabular}{lrrr}
\hline \hline \multicolumn{1}{c}{ Object } & $M_{V}{ }^{\mathrm{a}}$ & $N_{\mathrm{DC}}{ }^{\mathrm{b}}$ & $S_{\mathrm{DC}}$ \\
\hline Carina & -9.3 & 315 & 73 \\
Fornax & -13.2 & 97 & 0.6 \\
LMC & -18.5 & 2323 & $0.1^{\mathrm{c}}$ \\
\hline NGC 288 & -6.75 & 6 & 15 \\
NGC4372 & -7.79 & 8 & 10 \\
NGC5053 & -6.76 & 5 & 13 \\
NGC5139 ( $\omega$ Cen) & -10.26 & 34 & 3 \\
NGC5466 & -6.98 & 9 & 19 \\
NGC5904 (M5) & -8.81 & 5 & 2 \\
NGC6809 (M55) & -7.57 & 27 & 33 \\
\hline
\end{tabular}

${ }^{a}$ Values for Carina and Fornax from Mateo (1998), LMC from Binney \& Merrifield (1998), and globular clusters from the online (2010) version of Harris (1996) cata$\log$.

${ }^{\mathrm{b}}$ The values of $N_{\mathrm{DC}}$ for Carina and Fornax have been corrected to include all stars within the tidal King radius (see text). No correction was applied to the LMC or the globular clusters

${ }^{\mathrm{c}}$ Uncertain 